UWO-TH-06/04

\title{
Higher derivative corrections to near-extremal black holes in type IIB supergravity
}

\author{
Alex Buchel \\ Department of Applied Mathematics \\ University of Western Ontario \\ London, Ontario N6A 5B7, Canada \\ Perimeter Institute for Theoretical Physics \\ Waterloo, Ontario N2J 2W9, Canada
}

\begin{abstract}
We discuss string theory $\alpha^{\prime}$ corrections to charged near-extremal black 3-branes/black holes in type IIB supergravity. We find that supersymmetric global $A d S_{5} \times S^{5}$ geometry is not corrected to leading order in $\alpha^{\prime}$, while charged or non-extremal black 3-branes/black hole geometries receive $\alpha^{\prime}$ corrections. Following gauge theory-string theory correspondence the thermodynamics of these geometries is mapped to the thermodynamics of large- $n_{c} \mathcal{N}=4$ supersymmetric Yang-Mills theory at finite (large) 't Hooft coupling with the $U(1)_{R}$-charge chemical potential. We use holographic renormalization to compute the Gibbs free energy and the ADM mass of the near-extremal solutions. The remaining thermodynamic potentials are evaluated enforcing the first law of thermodynamics. We present analytic expressions for the $\alpha^{\prime}$ corrected thermodynamics of black holes in $A d S_{5} \times S^{5}$ and the thermodynamics of charged black 3-branes with identical chemical potentials for $\left[U(1)_{R}\right]^{3}$ charges and large (compare to chemical potential) temperature. We compute $\alpha^{\prime}$ corrections to Hawking-Page phase transition. We find that for nonzero chemical potential thermodynamics of near-extremal black 3-brane solution receives $\ln T$ correction to leading order in $\alpha^{\prime}$.
\end{abstract}

April 2006 


\section{Introduction}

Gauge theory-string theory correspondence of Maldacena [1,2] presents analytical tools to study non-perturbative aspects of strongly coupled gauge theories. In particular, by mapping finite temperature thermodynamics of gauge theories to thermodynamics of black holes in asymptotically anti-de-Sitter geometries it allows to use field theoretic intuition to uncover new phenomena in black hole physics. The most studied example of the gauge theory/string theory correspondence is that between $\mathcal{N}=4$ supersymmetric $S U\left(n_{c}\right)$ Yang-Mills (SYM) and $A d S_{5} \times S^{5}$ geometry in type IIB string theory. On the string theory side the latter duality is the simplest in the 't Hooft ( large $n_{c}$ ) limit and for large values of the 't Hooft coupling $\lambda=n_{c} g_{Y M}^{2} \gg 1$. In this regime both the string loop corrections $\propto \frac{1}{n_{c}}$ and the string theory $\alpha^{\prime}$ corrections $\propto \lambda^{-1 / 2}$ are suppressed and the supergravity approximation is reliable. Introducing finite temperature on the YangMills side corresponds to introducing horizon ( study nonextremal black hole solutions ) in asymptotic $A d S_{5} \times S^{5}$ type IIB supergravity. On the supergravity side one can study black holes with flat spatial horizon $\kappa=0$ ( these are often referred to as black branes ), or with spatial curvature $\kappa=\{-1,1\}$. The first example is a supergravity dual to a finite temperature $\mathcal{N}=4 \mathrm{SYM}$ on a space-time four-manifold $\mathcal{M}_{4} \equiv R^{3,1}$ originally studied in [3]. The other examples correspond to finite temperature $\mathcal{N}=4$ SYM on space-time manifolds $\mathcal{M}_{4} \equiv R \times A d S_{3}[4,5]$ and $\mathcal{M}_{4} \equiv R \times S_{3}$ correspondingly. Here, the existence of the second energy scale in the problem opens up a possibility for interesting phase transitions. Indeed, in [6] it was explained that the thermal HawkingPage phase transition for $\kappa=1$ AdS black holes is mapped to a finite temperature confinement/deconfinement phase transition of the $\mathcal{N}=4$ SYM on $R \times S^{3}$.

Supersymmetric $\mathcal{N}=4$ Yang Mills theory has $S O(6)_{R}$ symmetry realized as a global symmetry of the dual supergravity solution. Thus one can generalize SYM thermodynamics by introducing chemical potentials for $[U(1)]^{3} \subset S O(6)$ charges of the R-symmetry. On the gravity side this corresponds to study Riessner-Nordstrom black holes in $D=5 \mathcal{N}=8$ gauged supergravity (the so-called STU models) [7] or rotating black hole solutions in type IIB supergravity [8]. Thermodynamics of such black holes has been extensively studied in [9-12].

In this paper we study string theory $\alpha^{\prime}$ corrections to thermodynamics of neutral and charged black holes in asymptotic $A d S_{5} \times S^{5}$ geometry of type IIB supergravity. Previously, only thermodynamics of neutral $\kappa=0$ black hole has been analyzed in 
the literature $^{1}[14,15]$. Furthermore, it was argued in [16] that consistency of the hydrodynamic description ${ }^{2}$ of $\mathcal{N}=4 \mathrm{SYM}$ at finite 't Hooft coupling provides a highly nontrivial consistency check on the $\alpha^{\prime}$ thermodynamics of the $\kappa=0$ neutral black holes.

The paper is organized as follows. In the next section we present type IIB supergravity solution representing the ten-dimensional uplift ${ }^{3}$ of the STU models [7]. In section 3 we discuss our conventions and set up computational framework. For technical reasons we consider only the cases of neutral nonextremal black holes with spatial curvature $\kappa=\{0,1\}$, or black holes with spatial curvature $\kappa=\{0,1\}$ and identical charges under $U(1)^{3} \subset S O(6)$ R-symmetry. In section 4 we present $\alpha^{\prime}$-corrected equations of motion for these supergravity geometries. We find a convenient gauge in which all these equations decouple, and can be solved analytically for any values of the temperature and the chemical potential, apart from the (decoupled) $S^{5}$ warp factor second order ordinary differential equation. The latter equation we managed to solve analytically only for neutral $\kappa=0$ black holes ${ }^{4}$, and for charged $\kappa=0$ black holes in the high temperature $T$ (compare to the chemical potential $\mu_{q}$ ) approximation, i.e., $T \gg \mu_{q}$. In section 5 we consider thermodynamics of the $\alpha^{\prime}$ corrected nonextremal solutions. We discuss relevant aspects of $\alpha^{\prime}$ holographic renormalization and study thermodynamics of the $\kappa=\{0,1\}$ neutral black holes. Even though we do not have explicit analytic solution for the $S^{5}$ warp factor for nonextremal black holes with spatial curvature $\kappa=1$, we managed to compute analytically the Helmholtz free energy $F$ and the ADM mass $E$ of the black holes. Turns out, the neutral black hole entropy computed from the thermodynamic relation $S=(E-F) / T$ automatically satisfied the first law of thermodynamics. We find that the extremal global $A d S_{5} \times S^{5}$ geometry does not receive $\alpha^{\prime}$ corrections to leading order. We evaluate leading $\alpha^{\prime}$ corrections to the Hawking-Page transition temperature, or equivalently, leading 't Hooft coupling corrections to the finite temperature confinement-deconfinement phase transition of $\mathcal{N}=4$ supersymmetric Yang-Mills theory on $S^{3}$. Finally, we discuss thermodynamics of charged black holes. Here, the knowledge of the $S^{5}$ warp factor of the underlying geometry is crucial. We managed to find analytic solution only for $\kappa=0$ charged black

\footnotetext{
${ }^{1} \alpha^{\prime}$-corrected thermodynamics of near-extremal $(D 1, D 3)$ bound states was discussed in [13].

${ }^{2}$ Shear viscosity of the strongly coupled $\mathcal{N}=4$ SYM at finite 't Hooft coupling was originally computed in [17].

${ }^{3}$ We correct some misprints in [8].

${ }^{4}$ This case was previously considered in [15].
} 
holes to leading order in $\frac{\mu_{q}}{T} \ll 1$. We use holographic renormalization to compute the Gibbs free energy and the ADM mass of the black hole, and evaluate remaining thermodynamic properties ( i.e., the entropy, the physical R-symmetry charges and the conjugate chemical potentials) enforcing the first law of thermodynamics. Rather surprisingly, we find that thermodynamics of charged $\kappa=0$ black holes receives to leading order in $\alpha^{\prime}$ correction $\propto \ln \frac{T}{\epsilon}$. We comment on the appearance of an arbitrary energy scale $\epsilon$ in the system in the conclusion section. Some particularly technical details can be found in Appendix.

\section{Embedding STU models in type IIB SUGRA}

The ten dimensional lift of STU models [7] was explained in [8]. Of course, such uplifted solutions are fundamental to construction of $\alpha^{\prime}$-corrected black holes discuss in this paper. For this reason we write down explicitly corresponding 10d supergravity solutions fixing some misprints in [8].

The ten dimensional metric of a black hole with a spatial horizon curvature $\kappa$, the nonextremality parameter $\mu$, and charges $\tilde{q}_{i}, i=1,2,3$ under the Cartan subgroup $[U(1)]^{3}$ of the $S O(6)$ R-symmetry takes the form

$$
\begin{aligned}
d s_{10}^{2}= & \sqrt{\triangle}\left[-\left(H_{1} H_{2} H_{3}\right)^{-1} f d t^{2}+\left(f^{-1} d r^{2}+r^{2}\left(d \mathcal{M}_{3}\right)^{2}\right)\right] \\
& +\frac{1}{\sqrt{\triangle}} \sum_{i=1}^{3} L^{2} H_{i}\left(d \mu_{i}^{2}+\mu_{i}^{2}\left[d \phi_{i}+a_{i} d t\right]^{2}\right)
\end{aligned}
$$

where $\mathcal{M}_{3}=\left\{R^{3}, S^{3}\right\}$ is a spatial manifold corresponding to curvatures $\kappa=\{0,1\}$,

$$
\begin{aligned}
& a_{i}=\frac{\tilde{q}_{i}}{q_{i}} L^{-1}\left(H_{i}^{-1}-1\right), \quad H_{i}=1+\frac{q_{i}}{r^{2}}, \\
& \triangle=H_{1} H_{2} H_{3} \sum_{i=1}^{3} \frac{\mu_{i}^{2}}{H_{i}}, \quad f=\kappa-\frac{\mu}{r^{2}}+\frac{r^{2}}{L^{2}} H_{1} H_{2} H_{3},
\end{aligned}
$$

and

$$
\mu_{1}=\cos \theta_{1}, \quad \mu_{2}=\sin \theta_{1} \cos \theta_{2}, \quad \mu_{3}=\sin \theta_{1} \sin \theta_{2} .
$$

The physical charges $\tilde{q}_{i}$ are related to charge parameters $q_{i}$ as

$$
\tilde{q}_{i}=\sqrt{q_{i}\left(\mu+\kappa q_{i}\right)} .
$$

Finally, the dilaton is constant, and the five form is given by

$$
F_{5}=\mathcal{F}_{5}+\star \mathcal{F}_{5}, \quad \mathcal{F}_{5}=d B_{4},
$$


with

$$
B^{(4)}=-\frac{r^{4}}{L} \triangle d t \wedge d v o l_{\mathcal{M}_{3}}-L \sum_{i=1}^{3} \tilde{q}_{i} \mu_{i}^{2}\left(L d \phi_{i}-\frac{q_{i}}{\tilde{q}_{i}} d t\right) \wedge d v o l_{\mathcal{M}_{3}},
$$

where $d v o l_{\mathcal{M}_{3}}$ is a volume form on $\mathcal{M}_{3}$.

Without loss of generality we can set $L=1$. Computing $\alpha^{\prime}$ corrections for the most general 3-charge black holes is rather complicated. In this paper we consider only the following special cases:

- $\kappa=\{0,1\}$ and $q_{i}=0, i=1,2,3$;

- $\kappa=\{0,1\}$ and $q_{i}=Q, i=1,2,3$.

Let $r=r_{+}$be the position of the outer horizon of the black hole, i.e., the largest positive root of

$$
f\left(r_{+}\right)=0 .
$$

Neutral black holes have the following thermodynamic properties

$$
\begin{aligned}
& \pi T=\frac{\mu}{2 r_{+}^{3}}+\frac{r_{+}}{2}, \quad S=\frac{n_{c}^{2}}{2 \pi} r_{+}^{3}, \\
& E=\frac{n_{c}^{2}}{8 \pi^{2}}\left(3 \mu+\frac{3}{4} \kappa^{2}\right), \quad F=\frac{n_{c}^{2}}{8 \pi^{2}}\left(-\mu+\frac{3}{4} \kappa^{2}+2 r_{+}^{2} \kappa\right),
\end{aligned}
$$

where $T$ is the Hawking temperature, $S$ is the entropy density, $E$ is the energy density, and $F$ is the Helmholtz free energy density. Also, the Hawking-Page phase transition occurs at temperature $T_{H P}$ when the black hole free energy equals the ADM mass of the extremal $A d S_{5} \times S^{5}$ geometry [6]

$$
F(\mu)=E(\mu=0) \quad \Rightarrow \quad \pi T_{H P}=\frac{3}{2} \kappa^{1 / 2} .
$$

For the charged black holes we have $[9,12]$

$$
\begin{aligned}
& \pi T=\frac{\mu}{2 r_{+}^{3}}+\frac{r_{+}}{2} \frac{r_{+}^{2}-3 Q}{r_{+}^{2}-Q}, \quad S=\frac{n_{c}^{2}}{2 \pi} r_{+}^{3} \\
& E=\frac{n_{c}^{2}}{8 \pi^{2}}\left(3 \mu+\frac{3}{4} \kappa^{2}+6 Q \kappa\right), \quad \Omega=\frac{n_{c}^{2}}{8 \pi^{2}}\left(-\mu+\frac{3}{4} \kappa^{2}+2 r_{+}^{2} \kappa-2 Q \kappa\right), \\
& \mu_{q}=\frac{n_{c}^{2}}{4 \pi^{2} r_{+}^{2}}\left(\mu Q+\kappa Q^{2}\right)^{1 / 2}, \quad \tilde{Q}=\sqrt{\kappa Q^{2}+\mu Q},
\end{aligned}
$$

where $\Omega$ is the Gibbs free energy density, $\mu_{q}$ is the chemical potential corresponding to a physical charge density $\tilde{Q}$. Note that because all $U(1)$ R-symmetry charges are the same, the basic thermodynamic relation takes form

$$
\Omega=E-T S-3 \mu_{q} \tilde{Q}
$$


while the first law of thermodynamics is

$$
d \Omega=-S d T-3 \tilde{Q} d \mu_{q}
$$

\section{Computational framework}

We start with the tree level type IIB low-energy effective action in ten dimensions taking into account the leading order string corrections ${ }^{5}[18-21]$

$$
\begin{gathered}
I=\frac{1}{16 \pi G_{10}} \int_{\mathcal{M}_{10}} d^{10} x \sqrt{-g}\left[R_{10}-\frac{1}{2}(\partial \phi)^{2}-\frac{1}{4 \cdot 5 !}\left(F_{5}\right)^{2}+\ldots+\gamma e^{-\frac{3}{2} \phi} W+\ldots\right] \\
\gamma=\frac{1}{8} \zeta(3)\left(\alpha^{\prime}\right)^{3}
\end{gathered}
$$

where

$$
W=C^{h m n k} C_{p m n q} C_{h}^{r s p} C_{r s k}^{q}+\frac{1}{2} C^{h k m n} C_{p q m n} C_{h}^{r s p} C_{r s k}^{q} .
$$

As in $[14,17]$ we assume that in a chosen scheme self-dual $F_{5}$ form does not receive order $\left(\alpha^{\prime}\right)^{3}$ corrections. In [16] it was shown that such assumption leads to consistent description of hydrodynamic fluctuations of the $\kappa=0$ nonextremal black holes, which following gauge theory-string theory correspondence describes transport properties of strongly coupled finite temperature $\mathcal{N}=4$ Yang-Mills plasma at finite 't Hooft coupling. Specifically, the shear diffusion pole of the two-point correlation function of plasma stress-energy tensor is insensitive to the matter part (flux terms) in effective action (3.1) (for a general discussion see [22]). On the other hand, the sound pole in the stress-energy two-point correlation function depends on the matter content of the gravitational action (compare [23] and [24]), and thus is expected to be sensitive to the $\left(\alpha^{\prime}\right)^{3}$ structure of the flux terms in (3.1). Now, the shear viscosity computed from the diffusive pole must agree with the shear viscosity extracted from the attenuation of the sound waves. This agreement of both quantities derived from a specific form of the $\alpha^{\prime}$-corrected effective action (3.1) has been emphasized in $[16]^{6}$.

We represent ten dimensional background geometry describing $\gamma$-corrected charged

\footnotetext{
${ }^{5}$ In (3.1) ellipses stand for other fields not essential for the present analysis.

${ }^{6}$ It would be interesting to test consistency of black hole thermodynamics and hydrodynamics in $\alpha^{\prime}$-corrected type IIB supergravity effective action advocated in [25-27].
} 
black holes by the following ansatz

$$
\begin{aligned}
d s_{10}^{2}= & -D_{1}^{2} d t^{2}+D_{2}^{2} \frac{1}{\kappa}\left(d S^{3}\right)^{2}+D_{3}^{2} d r^{2}+D_{4}^{2} d \theta_{1}^{2}+D_{4}^{2} \sin ^{2} \theta_{1} d \theta_{2}^{2} \\
& +D_{4}^{2} \sum_{i=1}^{3} \mu_{i}^{2}\left(d \phi_{i}+D a d t\right)^{2}
\end{aligned}
$$

where $D_{i}=D_{i}\left(r, \theta_{1}, \theta_{2}\right)$ and $D a=D a\left(r, \theta_{1}, \theta_{2}\right), \mu_{i}$ are given by $(2.3)$, and $\left(d S^{3}\right)^{2}$ is a metric on a round three-sphere of unit radius. Taking $\kappa \rightarrow 0$ limit in (3.4) we obtain charged (or in ten dimensions rotating ) black hole solution with $\kappa=0$ spatial curvature of the horizon. Notice that in writing the metric ansatz (3.4) we assumed that $[U(1)]^{3}$ isometry is not broken by $\alpha^{\prime}$ corrections, also we assumed that our black hole carries identical charges under these $U(1)$ symmetries. We further assume that the dilaton $\phi=\phi\left(r, \theta_{1}, \theta_{2}\right)$. Since in the supergravity approximation $\left.\phi\right|_{\alpha^{\prime}=0}=0$ we see that $\phi \propto \gamma$, thus, as in [14], to leading order in $\gamma$ the metric deformations and the dilaton would decouple.

Next, we would like to obtain effective action involving supergravity modes $D_{i}, D a$, and $\phi$. To achieve this ${ }^{7}$, one needs to evaluate the $\alpha^{\prime}$ string corrected action (3.1) on the metric ansatz (3.4). The latter is a straightforward though a tedious exercise for a ten dimensional Ricci scalar and the fourth order Weyl tensor invariant $(3.3)^{8}$

$$
\begin{aligned}
& R_{10}=R_{10}\left[D_{i}\left(r, \theta_{1}, \theta_{2}\right), \operatorname{Da}\left(r, \theta_{1}, \theta_{2}\right),\left\{\theta_{1}, \theta_{2}\right\}\right], \\
& W=W\left[D_{i}\left(r, \theta_{1}, \theta_{2}\right), \operatorname{Da}\left(r, \theta_{1}, \theta_{2}\right),\left\{\theta_{1}, \theta_{2}\right\}\right] .
\end{aligned}
$$

A special care has to be taken with the five-form contribution in (3.1) [30]. In the latter case we find that integrating out the 5 -form one gets a contribution

$$
-\frac{1}{4 \cdot 5 !}\left(F_{5}\right)^{2}=-\frac{4 Q(\mu+\kappa Q)}{D_{4}^{4} D_{2}^{6}}-\frac{8}{D_{4}^{10}} .
$$

We separate 3-dimensional effective action describing $\gamma$-corrected metric derived from (3.1) into contribution coming from purely supergravity part and the $\gamma$-correction

$$
S_{\text {eff }}=S_{S U G R A}+S_{\gamma},
$$

\footnotetext{
${ }^{7}$ Effective low-dimensional supergravity actions derived in this way were recently used in $[28,29]$.

${ }^{8}$ Because of their complexity, we do not present explicit expressions for (3.5).
} 
with

$$
\begin{aligned}
S_{\text {SUGRA }}= & \frac{\operatorname{vol}\left(S^{3}\right)}{\kappa^{3 / 2}} \frac{(2 \pi)^{3}}{16 \pi G_{10}} \int d t \times \int_{0}^{\pi / 2} d \theta_{1} \int_{0}^{\pi / 2} d \theta_{2} \int_{r_{+}}^{\infty} d r \sin ^{3} \theta_{1} \cos \theta_{1} \sin \theta_{2} \cos \theta_{2} \\
& \times D_{1} D_{2}^{3} D_{3} D_{4}^{5}\left\{R_{10}-\frac{4 Q(\mu+\kappa Q)}{D_{4}^{4} D_{2}^{6}}-\frac{8}{D_{4}^{10}}\right\},
\end{aligned}
$$

and

$$
\begin{aligned}
S_{\gamma}= & \frac{\operatorname{vol}\left(S^{3}\right)}{\kappa^{3 / 2}} \frac{(2 \pi)^{3}}{16 \pi G_{10}} \int d t \times \int_{0}^{\pi / 2} d \theta_{1} \int_{0}^{\pi / 2} d \theta_{2} \int_{r_{+}}^{\infty} d r \sin ^{3} \theta_{1} \cos \theta_{1} \sin \theta_{2} \cos \theta_{2} \\
& \times D_{1} D_{2}^{3} D_{3} D_{4}^{5}\{\gamma W\} .
\end{aligned}
$$

In (3.8) and (3.9) $r_{+}$is the position of the outer horizon of metric (3.4) determined as the largest positive root of

$$
D_{1}\left(r_{+}, \theta_{1}, \theta_{2}\right)=0
$$

We find that even including $\alpha^{\prime}$ corrections $r_{+}$is actually independent of $\theta_{i}$. Lastly, the (decoupled to leading order in $\gamma$ ) equation for the dilaton reads

$$
\square \phi=\gamma \frac{3}{2} W
$$

where both the Dalambertian and the fourth order Weyl tensor invariant can be evaluated in $\alpha^{\prime}$ uncorrected metric (2.1).

Effective action (3.7) provides a consistent "Kaluza-Klein" reduction of (3.1) on $R \times \mathcal{M}_{3} \times\left[S^{1}\right]^{3}$ (along the time direction, world-volume $R^{3}$ or $S^{3}$ directions, and $[U(1)]^{3}$ isometries parameterized by $\phi_{i}$ ) in a sense that any solution of (3.7) is a solution of (3.1) [28]. Equations of motion derived from (3.7) take the form

$$
\begin{aligned}
& 0=\frac{\delta S}{\delta D_{i}}+\gamma J_{i}, \quad 0=\frac{\delta S}{\delta D a}+\gamma J_{a}, \\
& \gamma J_{i} \equiv \frac{\delta S_{\gamma}}{\delta D_{i}}, \quad \gamma J_{a} \equiv \frac{\delta S_{\gamma}}{\delta D a}
\end{aligned}
$$

Explicit evaluation of (3.12) is the most computationally consuming part of our framework. Again, due to their complexity, explicit expressions for (3.12) will not be presented here. 
To order $\gamma^{0}(3.12)$ can be solved with (in agreement with (2.1))

$$
\begin{aligned}
& D_{1}^{(0)}=\frac{r^{2}}{r^{2}+Q}\left(\kappa-\frac{\mu}{r^{2}}+r^{2}\left(1+\frac{Q}{r^{2}}\right)^{3}\right)^{1 / 2}, \quad D_{2}^{(0)}=\left(r^{2}+Q\right)^{1 / 2}, \\
& D_{3}^{(0)}=\left(1+\frac{Q}{r^{2}}\right)^{1 / 2}\left(\kappa-\frac{\mu}{r^{2}}+r^{2}\left(1+\frac{Q}{r^{2}}\right)^{3}\right)^{-1 / 2}, \quad D_{4}^{(0)}=1, \\
& D a^{(0)}=-\frac{\left(Q \mu+\kappa^{2} Q\right)^{1 / 2}}{r^{2}+Q} .
\end{aligned}
$$

To order $\gamma^{1}$ we find it convenient to choose a radial coordinate so that

$$
\begin{aligned}
& D_{1}\left(r, \theta_{1}, \theta_{2}\right)=D_{1}^{(0)} \times\left(1+\gamma\left(A+B-\frac{5}{3} \nu\right)\right), \\
& D_{2}\left(r, \theta_{1}, \theta_{2}\right)=D_{2}^{(0)} \times\left(1+\gamma\left(A-\frac{5}{3} \nu\right)\right), \\
& D_{3}\left(r, \theta_{1}, \theta_{2}\right)=D_{3}^{(0)} \times\left(1+\gamma\left(2 A-B-\frac{5}{3} \nu\right)\right), \\
& D_{4}\left(r, \theta_{1}, \theta_{2}\right)=D_{4}^{(0)} \times(1+\gamma \nu), \quad D a\left(r, \theta_{1}, \theta_{2}\right)=D a^{(0)} \times(1+\gamma a) .
\end{aligned}
$$

Upon deriving equations of motion (3.12) in their generality, we find that it is consistent to assume that deformations $A, B, \nu, a$ are functions of the radial coordinate only. In other words, given (3.14) and (3.12) we obtain second order system of ordinary differential equations for $\{A, B, \nu, a\}$. The advantage of fixing radial coordinate to order $\gamma$ as in (3.14) is that differential equations for each deformation factor decouple with such a choice.

We conclude this section by specifying the boundary conditions for $\{A, B, \nu, a\}$ :

$$
\begin{aligned}
& \{A, B, \nu, a\} \quad \text { are regular functions as } \quad r \rightarrow r_{+}+0, \\
& \{A, B, \nu, a\} \rightarrow 0 \quad \text { as } \quad r \rightarrow \infty .
\end{aligned}
$$

\section{$4 \alpha^{\prime}$-correct black hole solutions in asymptotic $A d S_{5} \times S^{5}$ type IIB supergravity}

In this section we present differential equations (and their) solutions describing $\alpha^{\prime}$ corrections to neutral and charged black holes with $\kappa=\{0,1\}$ spatial curvature of the horizon in asymptotic $A d S_{5} \times S^{5}$ type IIB supergravity. The equations are obtained from (3.12) within ansatz (3.14). We find that solutions to these equations subject to 
boundary conditions (3.15) are unique. It is simplest to write these equations using a new radial coordinate

$$
x \equiv r^{2}+Q
$$

We also denote

$$
x_{+} \equiv r_{+}^{2}+Q
$$

\subsection{Neutral black hole with horizon spatial curvature $\kappa$}

In this case the decoupled system of equations is the simplest. We find ${ }^{9}$

$$
\begin{gathered}
0=A^{\prime \prime}+\frac{360 \mu^{3}}{x^{8}}, \\
0=B^{\prime \prime}+\frac{9 x^{2}-\mu+5 \kappa x}{2 x\left(x^{2}+\kappa x-\mu\right)} B^{\prime}+\frac{12 x^{2}-2 \mu+7 \kappa x}{2 x\left(x^{2}+\kappa x-\mu\right)} A^{\prime}+\frac{6 x+\kappa}{2 x\left(x^{2}+\kappa x-\mu\right)} B \\
-\frac{12 x+\kappa}{2 x\left(x^{2}+\kappa x-\mu\right)} A-\frac{5 \mu^{3}\left(-639 \mu+608 \kappa x+576 x^{2}\right)}{2 x^{8}\left(x^{2}+\kappa x-\mu\right)}, \\
0=\nu^{\prime \prime}+\frac{2 \kappa x-\mu+3 x^{2}}{x\left(x^{2}+\kappa x-\mu\right)} \nu^{\prime}-\frac{8}{x^{2}+\kappa x-\mu} \nu+\frac{135 \mu^{4}}{8 x^{8}\left(x^{2}+\kappa x-\mu\right)} .
\end{gathered}
$$

Additionally, there is a first order constrain arising from reparametrization invariance of the radial coordinate

$$
0=B^{\prime}+\frac{4 x^{2}-2 \mu+3 \kappa x}{x^{2}+\kappa x-\mu} A^{\prime}+\frac{\kappa+2 x}{x^{2}+\kappa x-\mu} B-\frac{\kappa+4 x}{x^{2}+\kappa x-\mu} A+\frac{5 \mu^{3}(16 \kappa x-27 \mu)}{x^{7}\left(x^{2}+\kappa x-\mu\right)} .
$$

It is straightforward to verify that (4.6) is consistent with (4.3) and (4.4). We can explicitly solve for $A$ and $B$ subject to boundary conditions (3.15)

$$
\begin{gathered}
A=-\frac{60 \mu^{3}}{7 x^{6}} \\
B=\frac{\mu^{3}}{x^{2}+\kappa x-\mu}\left(\frac{60}{x^{4}}+\frac{340 \kappa}{7 x^{5}}-\frac{555 \mu}{14 x^{6}}+\mathcal{B}\right),
\end{gathered}
$$

where

$$
\mathcal{B}=-\frac{60}{x_{+}^{4}}-\frac{340 \kappa}{7 x_{+}^{5}}+\frac{555 \mu}{14 x_{+}^{6}}
$$

\footnotetext{
${ }^{9}$ The prime denotes derivative with respect to $x$.
} 
Unfortunately, we were unable to solve (4.5) apart from $\kappa=0$ case. In the latter case we reproduce the result of $[15]$

$$
\left.\nu\right|_{\kappa=0} \equiv \nu_{0}=\frac{15 \mu^{2}\left(\mu+x^{2}\right)}{32 x^{6}} .
$$

Notice that in the limit $\kappa \rightarrow 0$ and with the radial coordinate reparametrization

$$
r \rightarrow r\left(1+\gamma \frac{60 r_{+}^{12}}{7 r^{12}}\right) \quad \text { and } \quad r_{+} \rightarrow r_{+}\left(1+\gamma \frac{60}{7}\right)
$$

our solution reproduces the known $\alpha^{\prime}$-corrected geometry of the near-extremal flat D3-branes $[14,15]$. In is straightforward to analyze equation (4.5) and observe that the absence of the singularity at $x=x_{+}$along with the ultraviolet (UV) boundary conditions $\nu \rightarrow 0$ as $x \rightarrow \infty$, uniquely determine the $S^{5}$ warp factor $\nu(x)$. Turns out that for the thermodynamics of the neutral black holes we would only need the UV asymptotic of $\nu$ as $x \rightarrow \infty$

$$
\nu(x) \sim \mathcal{O}\left(x^{-4}\right) .
$$

For completeness we write down the solution to the dilaton equation (3.11)

$$
\phi=\frac{45 \mu^{4}}{4} \int_{x}^{\infty} \frac{d z}{z\left(z^{2}+\kappa z-\mu\right)}\left(\frac{1}{z^{6}}-\frac{1}{x_{+}^{6}}\right) .
$$

Integral in (4.13) can be evaluated explicitly. It is easy to see that the $\kappa=0$ limit reproduces the result of $[14,15]$.

We conclude this section with comments on the $\alpha^{\prime}$ corrections to global extremal $A d S_{5} \times S^{5}$ geometry. From previous work $[14,15]$ we know that the fourth order Weyl invariant (3.3) vanishing at the extremality in the Poincare patch. Since it is a local invariant, it must vanish in global coordinates as well. The latter implies that the global extremal $A d S_{5} \times S^{5}$ geometry does not receive $\alpha^{\prime}$ corrections $^{10}$. From (4.7), (4.8) we see that at the extremality, i.e., setting $\mu=0$

$$
\left.A\right|_{\mu=0}=0,\left.\quad B\right|_{\mu=0}=0 \text {. }
$$

Furthermore, the general solution of (4.5) at the extremality reads

$$
\begin{aligned}
\left.\nu\right|_{\mu=0}= & \mathcal{C}_{1}\left(3 \kappa^{2}+12 \kappa x+10 x^{2}\right) \\
& +\frac{\mathcal{C}_{2}}{x}\left(-3 x\left(3 \kappa^{2}+12 \kappa x+10 x^{2}\right) \ln \left(1+\frac{\kappa}{x}\right)+\kappa\left(\kappa^{2}+21 \kappa x+30 x^{2}\right) .\right.
\end{aligned}
$$

\footnotetext{
${ }^{10}$ Actually, Kallosh-Rajaraman arguments [31] are much more powerful than $\alpha^{\prime}$-leading order explicit computations and imply that global extremal $A d S_{5} \times S^{5}$ geometry does not receive $\alpha^{\prime}$ corrections at any order.
} 
Now, the appropriate boundary conditions is that $\nu$ is nonsingular as $x \rightarrow+0$, and vanishes as $x \rightarrow \infty$. Thus

$$
\left.\nu\right|_{\mu=0}=0 .
$$

\section{2 $\kappa=1$ charged black holes}

In this case we find

$$
\begin{aligned}
& 0=A^{\prime \prime}+J_{A}, \\
& 0=B^{\prime \prime}-\frac{2\left(\mu-2 x-3 x^{2}+2 Q\right)}{x^{2}-2 x Q+Q^{2}+x^{3}-\mu x+Q \mu} B^{\prime}+\frac{2(1+3 x)}{x^{2}-2 x Q+Q^{2}+x^{3}-\mu x+Q \mu} B \\
& -\frac{4\left(\mu-2 x-3 x^{2}+2 Q\right)}{x^{2}-2 x Q+Q^{2}+x^{3}-\mu x+Q \mu} A^{\prime}-\frac{2(1+6 x)}{x^{2}-2 x Q+Q^{2}+x^{3}-\mu x+Q \mu} A+J_{B} \text {, } \\
& 0=\nu^{\prime \prime}-\frac{\mu-2 x-3 x^{2}+2 Q}{x^{2}-2 x Q+Q^{2}+x^{3}-\mu x+Q \mu} \nu^{\prime}-\frac{4\left(Q^{2}+Q \mu+10 x^{3}\right)}{5 x^{2}\left(x^{2}-2 x Q+Q^{2}+x^{3}-\mu x+Q \mu\right)} \nu \\
& -\frac{3\left(Q^{2}+Q \mu+4 x^{3}-2 \mu x-4 x Q+3 x^{2}\right)}{5 x\left(x^{2}-2 x Q+Q^{2}+x^{3}-\mu x+Q \mu\right)} A^{\prime}-\frac{3}{5 x} B^{\prime} \\
& +\frac{3\left(Q^{2}+Q \mu+x^{2}+4 x^{3}\right)}{5 x^{2}\left(x^{2}-2 x Q+Q^{2}+x^{3}-\mu x+Q \mu\right)} A+\frac{3\left(Q^{2}+Q \mu-2 x^{3}-x^{2}\right)}{5 x^{2}\left(x^{2}-2 x Q+Q^{2}+x^{3}-\mu x+Q \mu\right)} B \\
& +J_{\nu} \\
& 0=a^{\prime \prime}-\frac{16}{3 x} \nu^{\prime}+J_{a} .
\end{aligned}
$$

Additionally, there is a first order constraint arising from reparametrization invariance of the radial coordinate

$$
\begin{aligned}
& 0=\left(Q^{2}+Q \mu+4 x^{3}-2 \mu x-4 x Q+3 x^{2}\right) A^{\prime}+\left(x^{2}-2 x Q+Q^{2}+x^{3}-\mu x+Q \mu\right) B^{\prime} \\
& -\frac{Q(Q+\mu)}{3} a^{\prime}-\frac{Q^{2}+Q \mu+x^{2}+4 x^{3}}{x} A-\frac{Q^{2}+Q \mu-2 x^{3}-x^{2}}{x} B+\frac{16 Q(Q+\mu)}{9 x} \nu \\
& +\frac{Q(Q+\mu)}{3 x} a+J_{\text {const } .}
\end{aligned}
$$

Explicit expressions for the inhomogeneous sources $\left\{J_{A}, J_{B}, J_{\nu}, J_{a}, J_{\text {const }}\right\}$ are given in Appendix A. It is straightforward to verify that (4.21) is consistent with (4.17)-(4.20). 
Furthermore, equations (4.17)-(4.21) in the limit $Q \rightarrow 0$ reproduce equations (4.3)-(4.6) in the limit $\kappa \rightarrow 1$.

Solution of (4.17) with boundary conditions (3.15) is

$$
\begin{aligned}
A= & \frac{27569 Q^{3}}{360 x^{9}}\left(\mu^{3}+Q^{3}+3 \mu Q^{2}+3 \mu^{2} Q\right)-\frac{21049 Q^{2}}{216 x^{8}}\left(\mu^{3}+2 Q^{3}+5 \mu Q^{2}+4 \mu^{2} Q\right) \\
& +\frac{Q}{112 x^{7}}\left(5359 \mu^{3}+23799 \mu^{2} Q+36880 \mu Q^{2}+18440 Q^{3}\right)+\frac{1}{x^{6}}\left(-\frac{320}{7} Q^{3}+\frac{38}{21} \mu^{2} Q^{2}\right. \\
& \left.+\frac{38}{21} Q^{4}-\frac{480}{7} \mu Q^{2}-\frac{60}{7} \mu^{3}-40 \mu^{2} Q+\frac{76}{21} Q^{3} \mu\right)-\frac{4 Q}{9 x^{5}}\left(2 Q^{2}+3 Q \mu+\mu^{2}\right) .
\end{aligned}
$$

The most general solution of (4.18) is

$$
\begin{aligned}
& B=\frac{x \mathcal{B}_{1}+\mathcal{B}_{2}}{x^{3}+x^{2}-(\mu+2 Q) x+Q(Q+\mu)}+\frac{1}{15120 x^{9}\left(x^{3}+x^{2}-(\mu+2 Q) x+Q(Q+\mu)\right)} \\
& \times\left(-302400 Q(Q+\mu)(2 Q+\mu) x^{7}+\left(554400 Q^{4}+4005120 Q^{3}+1108800 Q^{3} \mu\right.\right. \\
& \left.+554400 \mu^{2} Q^{2}+6007680 \mu Q^{2}+3816960 \mu^{2} Q+907200 \mu^{3}\right) x^{6}+\left(-14110176 Q^{4}\right. \\
& -28220352 Q^{3} \mu+3594240 Q^{3}+5391360 \mu Q^{2}-18524976 \mu^{2} Q^{2}+3265920 \mu^{2} Q \\
& \left.-4414800 \mu^{3} Q+734400 \mu^{3}\right) x^{5}+\left(16345800 Q^{5}+40864500 Q^{4} \mu-18626040 Q^{4}\right. \\
& -37252080 Q^{3} \mu+32691600 Q^{3} \mu^{2}-26330625 \mu^{2} Q^{2}+8172900 \mu^{3} Q^{2}-7704585 \mu^{3} Q \\
& \left.-599400 \mu^{4}\right) x^{4}-10 Q(Q+\mu)\left(629580 Q^{4}+1259160 Q^{3} \mu-3870674 Q^{3}-5806011 \mu Q^{2}\right. \\
& \left.+629580 \mu^{2} Q^{2}-2679043 \mu^{2} Q-371853 \mu^{3}\right) x^{3}-2 Q^{2}\left(20176669 Q^{2}+20176669 Q \mu\right. \\
& \left.\left.+4556605 \mu^{2}\right)(Q+\mu)^{2} x^{2}+10549266 Q^{3}(2 Q+\mu)(Q+\mu)^{3} x-4423125 Q^{4}(Q+\mu)^{4}\right),
\end{aligned}
$$

where $\mathcal{B}_{i}$ are integration constants. Given (4.22) and (4.23) we can determine $a(x)$ 
from (4.21)

$$
\begin{aligned}
a & =\frac{3 \mathcal{B}_{2}}{Q(Q+\mu)}+x\left(\mathcal{C}_{a}+\frac{16}{3} \int_{x_{+}}^{x} d z \frac{\nu(z)}{z^{2}}\right)-\frac{1}{2520 x^{9}}\left(-60480 Q(Q+\mu) x^{6}\right. \\
& +\left(628992 Q^{2}+241920 \mu^{2}+628992 Q \mu\right) x^{5}+\left(564480 Q \mu-1404480 Q^{3}-2106720 \mu Q^{2}\right. \\
& \left.-702240 \mu^{2} Q+564480 Q^{2}+201600 \mu^{2}\right) x^{4}+\left(-2093760 Q^{3}+763920 Q^{4}-151200 \mu^{3}\right. \\
& \left.+763920 \mu^{2} Q^{2}-3140640 \mu Q^{2}-1349280 \mu^{2} Q+1527840 Q^{3} \mu\right) x^{3} \\
& +315 Q(Q+\mu)\left(1909 \mu^{2}+9372 Q \mu+9372 Q^{2}\right) x^{2}-931000 Q^{2}(2 Q+\mu)(Q+\mu)^{2} x \\
& \left.+440622 Q^{3}(Q+\mu)^{3}\right)
\end{aligned}
$$

where $\mathcal{C}_{a}$ is a new integration constant. Since the boundary conditions are such that $a \rightarrow 0$ as $x \rightarrow \infty$, we conclude from (4.24) that

$$
\mathcal{B}_{2}=0, \quad \mathcal{C}_{a}=-\frac{16}{3} \int_{x_{+}}^{\infty} d z \frac{\nu(z)}{z^{2}} .
$$

The remaining integration constant $\mathcal{B}_{1}$ is fixed requiring that $B$ is nonsingular as $x \rightarrow x_{+}+0$

$$
\begin{aligned}
& \mathcal{B}_{1}=\frac{1}{15120 x_{+}^{10}}\left(302400 Q(2 Q+\mu)(Q+\mu) x_{+}^{7}+\left(-554400 Q^{4}-4005120 Q^{3}-907200 \mu^{3}\right.\right. \\
& \left.-1108800 Q^{3} \mu-6007680 \mu Q^{2}-3816960 \mu^{2} Q-554400 \mu^{2} Q^{2}\right) x_{+}^{6}+\left(-3594240 Q^{3}\right. \\
& -734400 \mu^{3}+14110176 Q^{4}+28220352 Q^{3} \mu+18524976 \mu^{2} Q^{2}-3265920 \mu^{2} Q \\
& \left.+4414800 \mu^{3} Q-5391360 \mu Q^{2}\right) x_{+}^{5}+\left(-16345800 Q^{5}+599400 \mu^{4}+18626040 Q^{4}\right. \\
& -40864500 \mu Q^{4}+7704585 \mu^{3} Q-8172900 \mu^{3} Q^{2}+37252080 Q^{3} \mu+26330625 \mu^{2} Q^{2} \\
& \left.-32691600 \mu^{2} Q^{3}\right) x_{+}^{4}-10 Q(Q+\mu)\left(371853 \mu^{3}+2679043 \mu^{2} Q-629580 \mu^{2} Q^{2}\right. \\
& \left.-1259160 Q^{3} \mu+5806011 \mu Q^{2}-629580 Q^{4}+3870674 Q^{3}\right) x_{+}^{3}+2 Q^{2}\left(4556605 \mu^{2}\right. \\
& \left.+20176669 Q \mu+20176669 Q^{2}\right)(Q+\mu)^{2} x_{+}^{2}-10549266 Q^{3}(2 Q+\mu)(Q+\mu)^{3} x_{+} \\
& \left.+4423125 Q^{4}(Q+\mu)^{4}\right) .
\end{aligned}
$$

The resulting equation for the $S^{5}$ warp factor $\nu(x)$ is

$$
\begin{aligned}
& 0=\nu^{\prime \prime}-\frac{\mu-2 x-3 x^{2}+2 Q}{x^{2}-2 x Q+Q^{2}+x^{3}-\mu x+Q \mu} \nu^{\prime}-\frac{4\left(Q^{2}+Q \mu+10 x^{3}\right)}{5 x^{2}\left(x^{2}-2 x Q+Q^{2}+x^{3}-\mu x+Q \mu\right)} \nu \\
& +\mathcal{I}_{\nu}
\end{aligned}
$$


where $\mathcal{I}_{\nu}$ is given in Appendix A. We were unable to find analytic solution to (4.27) even for $^{11} Q=0$. Nonetheless, it is straightforward to verify that requiring nonsingularity at the horizon and the vanishing of $\nu(x)$ as $x \rightarrow \infty$, the $S^{5}$ warp factor $\nu(x)$ is uniquely determined. As in (4.12)

$$
\nu(x) \sim \mathcal{O}\left(x^{-4}\right) .
$$

For completeness we present the dilaton equation

$$
0=\phi^{\prime \prime}-\frac{-3 x^{2}+\mu+2 Q-2 x}{-\mu x+x^{3}+Q^{2}-2 Q x+x^{2}+Q \mu} \phi^{\prime}+J_{\phi},
$$

where $J_{\phi}$ is given in Appendix A. The dilaton equation can be solved analytically.

\section{3 $\kappa=0$ charged black branes}

In this case we find

$$
\begin{gathered}
0=A^{\prime \prime}+J_{A}, \\
0=B^{\prime \prime}+\frac{2\left(3 x^{2}-\mu\right)}{-x \mu+Q \mu+x^{3}} B^{\prime}+\frac{6 x}{-x \mu+Q \mu+x^{3}} B+\frac{4\left(3 x^{2}-\mu\right)}{-x \mu+Q \mu+x^{3}} A^{\prime} \\
-\frac{12 x}{-x \mu+Q \mu+x^{3}} A+J_{B}, \\
0=\nu^{\prime \prime}+\frac{3 x^{2}-\mu}{-x \mu+Q \mu+x^{3}} \nu^{\prime}-\frac{4\left(Q \mu+10 x^{3}\right)}{5 x^{2}\left(-x \mu+Q \mu+x^{3}\right)} \nu-\frac{3\left(4 x^{3}+Q \mu-2 x \mu\right)}{5 x\left(-x \mu+Q \mu+x^{3}\right)} A^{\prime} \\
-\frac{3}{5 x} B^{\prime}+\frac{3\left(Q \mu+4 x^{3}\right)}{5 x^{2}\left(-x \mu+Q \mu+x^{3}\right)} A+\frac{3\left(Q \mu-2 x^{3}\right)}{5 x^{2}\left(-x \mu+Q \mu+x^{3}\right)} B+J_{\nu}, \\
0=a^{\prime \prime}-\frac{16}{3 x} \nu^{\prime}+J_{a} .
\end{gathered}
$$

Additionally, there is a first order constraint arising from reparametrization invariance of the radial coordinate

$$
\begin{aligned}
0= & \left(4 x^{3}+Q \mu-2 x \mu\right) A^{\prime}+\left(-x \mu+Q \mu+x^{3}\right) B^{\prime}-\frac{Q \mu}{3} a^{\prime}-\frac{Q \mu+4 x^{3}}{x} A \\
& -\frac{Q \mu-2 x^{3}}{x} B+\frac{16 \mu Q}{9 x} \nu+\frac{\mu Q}{3 x} a+J_{\text {const } .}
\end{aligned}
$$

Explicit expressions for the inhomogeneous sources $\left\{J_{A}, J_{B}, J_{\nu}, J_{a}, J_{\text {const }}\right\}$ are given in Appendix B. It is straightforward to verify that (4.34) is consistent with (4.30)-(4.33).

\footnotetext{
${ }^{11}$ In the case of $\kappa=0$ charged black holes the corresponding equation can be solved perturbatively in $Q$.
} 
Furthermore, equations (4.30)-(4.34) in the limit $Q \rightarrow 0$ reproduce equations (4.3)-(4.6) in the limit $\kappa \rightarrow 0$.

Solution of (4.30) with boundary conditions (3.15) is

$$
A=\frac{27569 \mu^{3} Q^{3}}{360 x^{9}}-\frac{21049 Q^{2} \mu^{3}}{216 x^{8}}+\frac{5359 Q \mu^{3}}{112 x^{7}}+\frac{2 \mu^{2}\left(19 Q^{2}-90 \mu\right)}{21 x^{6}}-\frac{4 Q \mu^{2}}{9 x^{5}} .
$$

The most general solution of (4.31) is

$$
\begin{aligned}
& B=\frac{x \mathcal{B}_{1}+\mathcal{B}_{2}}{x^{3}-\mu x+Q \mu}+\frac{1}{15120 x^{9}\left(x^{3}-\mu x+Q \mu\right)}\left(-4423125 \mu^{4} Q^{4}+10549266 Q^{3} \mu^{4} x\right. \\
& -9113210 Q^{2} \mu^{4} x^{2}-30\left(-123951 \mu+209860 Q^{2}\right) \mu^{3} Q x^{3}+8100\left(1009 Q^{2}-74 \mu\right) \mu^{3} x^{4} \\
& \left.-4414800 Q \mu^{3} x^{5}+50400 \mu^{2}\left(11 Q^{2}+18 \mu\right) x^{6}-302400 Q \mu^{2} x^{7}\right)
\end{aligned}
$$

where $\mathcal{B}_{i}$ are integration constants. Given (4.35) and (4.36) we can determine $a(x)$ from (4.34)

$$
\begin{aligned}
& a=\frac{3 \mathcal{B}_{2}}{Q \mu}+x\left(\mathcal{C}_{a}+\frac{16}{3} \int_{x_{+}}^{x} d z \frac{\nu(z)}{z^{2}}\right)-\frac{\mu}{2520 x^{9}}\left(440622 Q^{3} \mu^{2}-931000 Q^{2} \mu^{2} x\right. \\
& +601335 Q \mu^{2} x^{2}+\left(763920 \mu Q^{2}-151200 \mu^{2}\right) x^{3}-702240 Q \mu x^{4}+241920 \mu x^{5} \\
& \left.-60480 Q x^{6}\right)
\end{aligned}
$$

where $\mathcal{C}_{a}$ is a new integration constant. Since the boundary conditions are such that $a \rightarrow 0$ as $x \rightarrow \infty$, we conclude from (4.37) that

$$
\mathcal{B}_{2}=0, \quad \mathcal{C}_{a}=-\frac{16}{3} \int_{x_{+}}^{\infty} d z \frac{\nu(z)}{z^{2}}
$$

The remaining integration constant $\mathcal{B}_{1}$ is fixed requiring that $B$ is nonsingular as $x \rightarrow x_{+}+0$

$$
\begin{aligned}
& \mathcal{B}_{1}=\frac{\mu^{2}}{15120 x_{+}^{10}}\left(302400 Q x_{+}^{7}+\left(-554400 Q^{2}-907200 \mu\right) x_{+}^{6}+4414800 Q \mu x_{+}^{5}\right. \\
& -8100 \mu\left(1009 Q^{2}-74 \mu\right) x_{+}^{4}+30 Q \mu\left(-123951 \mu+209860 Q^{2}\right) x_{+}^{3}+9113210 Q^{2} \mu^{2} x_{+}^{2} \\
& \left.-10549266 Q^{3} \mu^{2} x_{+}+4423125 Q^{4} \mu^{2}\right) .
\end{aligned}
$$


The resulting equation for the $S^{5}$ warp factor $\nu(x)$ is

$$
0=\nu^{\prime \prime}+\frac{3 x^{2}-\mu}{-x \mu+Q \mu+x^{3}} \nu^{\prime}-\frac{4\left(Q \mu+10 x^{3}\right)}{5 x^{2}\left(-x \mu+Q \mu+x^{3}\right)} \nu+\mathcal{I}_{\nu},
$$

where $\mathcal{I}_{\nu}$ is given in Appendix B. We were unable to find analytic solution to (4.40) for general $Q$. In the next section we solve this equation analytically to leading order in $Q$. It is straightforward to verify that requiring nonsingularity at the horizon and the vanishing of $\nu(x)$ as $x \rightarrow \infty$, the $S^{5}$ warp factor $\nu(x)$ is uniquely determined. Again, as in (4.12)

$$
\nu(x) \sim \mathcal{O}\left(x^{-4}\right) .
$$

For completeness we present the dilaton equation

$$
0=\phi^{\prime \prime}-\frac{-3 x^{2}+\mu}{Q \mu+x^{3}-\mu x} \phi^{\prime}+J_{\phi},
$$

where $J_{\phi}$ is given in Appendix B. The dilaton equation can be solved analytically.

\subsection{1 $\kappa=0$ charge black branes at large temperature}

As we explain in Section 5, to analyze the thermodynamics of charged black holes one needs to know explicitly the value of the gauge potential $a(x)$ at the horizon, $x=x_{+}$. From (4.37), the latter implies that we need to know explicit expression for $\mathcal{C}_{a}$ (4.38). We were unable to compute $\mathcal{C}_{a}$ for arbitrary values of the nonextremality parameter $\mu$ and the charge parameter $Q$. In this section we evaluate $\mathcal{C}_{a}$ to leading order in $Q$. Physically, above approximation means that we would be interested in the thermodynamics of $\kappa=0$ charged black holes in the regime when the physical charge chemical potential $\mu_{q} \ll T$.

To leading order in $Q$ the solution to (4.40) which is nonsingular at the horizon $x=x_{+}$and vanishes as $x \rightarrow \infty$ is given by

$$
\begin{aligned}
\nu= & \nu_{0}+Q\left(-\frac{113241\left(2 x^{2}-\mu\right)}{5600 \mu^{3 / 2}}\left(\ln \left(1-\frac{\mu}{x^{2}}\right)+2 \operatorname{arctanh} \frac{\sqrt{\mu}}{x}\right)+\frac{9857 \mu^{3}}{3920 x^{7}}-\frac{1144277 \mu^{2}}{98000 x^{5}}\right. \\
& \left.+\frac{37747 \mu}{14000 x^{3}}-\frac{37747}{2800 x}-\frac{113241}{2800 \sqrt{\mu}}+\frac{113241 x}{1400 \mu}\right)+\mathcal{O}\left(Q^{2}\right),
\end{aligned}
$$

where $\nu_{0}$ is the $\kappa=0, Q=0$ black hole warp factor (4.10). Given (4.43) and using

$$
x_{+}=\sqrt{\mu}-\frac{1}{2} Q+\mathcal{O}\left(Q^{2}\right)
$$


we find ${ }^{12}$

$$
\mathcal{C}_{a}=-\frac{6}{7 \sqrt{\mu}}+\mathcal{O}(Q)
$$

For convenience, we collect all formulas for the $\kappa=0 \alpha^{\prime}$-corrected charged black hole to order $\mathcal{O}\left(Q^{2}\right)$ in Appendix C.

\section{$5 \quad$ Leading $\alpha^{\prime}$ corrections to thermodynamics of $A d S_{5} \times S^{5}$ black holes}

\subsection{General framework}

In this section we discuss the thermodynamics of the $\alpha^{\prime}$ corrected black holes in asymptotic $A d S_{5} \times S^{5}$ geometry of type IIB supergravity. We assume that the following two statements are correct even in the presence of $\alpha^{\prime}$ corrections:

- first, for neutral black holes we have a thermodynamic relation between Helmholtz free energy density $F$, the energy density $E$, the entropy density $S$ and the temperature $T$

$$
F=E-T S
$$

second, for charged black holes we have a thermodynamic relation between Gibbs free energy density $\Omega$, the energy density $E$, the entropy density $S$, the chemical potential $\mu_{q}$ for the true physical charge density $\tilde{Q}$, and the temperature $T$

$$
\Omega=E-T S-3 \mu_{q} \tilde{Q}
$$

where the factor 3 arises because we introduced identical chemical potential for all three charges;

- for the neutral black holes the first law of thermodynamics takes form

$$
d F=-S d T
$$

while for the charged black holes we have

$$
d \Omega=-S d T-3 \tilde{Q} d \mu_{q}
$$

Since we are going to rely on the validity of (5.1)-(5.4) in black hole thermodynamics, it is worthwhile recalling some nontrivial checks on the latter relations. In the

\footnotetext{
${ }^{12}$ We will need the gauge potential $a(x)$ only to order $\mathcal{O}\left(Q^{0}\right)$.
} 
absence of $\alpha^{\prime}$ corrections one can independently evaluate each quantity in (5.1) or (5.2) and verify (5.3) and (5.4). Indeed, one can use holographic renormalization [32-34] to compute the energy density (from the one-point correlation function of the holographic stress-energy tensor) and the Helmholtz ( or Gibbs) free energy density (as regularized Euclidean action for the background geometry). It is straightforward to compute Hawking temperature (surface gravity) of the black hole and the black hole entropy density (as a quarter of the horizon area measured in Planck units). Finally, the chemical potential can be determined from the value of the gauge field at the horizon [10], and the conserved physical charge densities can be determined by applying the Gauss's law. All this was verified in a variety of highly nontrivial examples ${ }^{13}$. The situation is much more complicate for black holes including $\alpha^{\prime}$ correction. To name one complication, the black hole entropy density is no longer determined simply by the area of the horizon. This was first observed imposing thermodynamic relation (5.1) and (5.3) for the $\kappa=0 \alpha^{\prime}$-corrected black holes in asymptotic $A d S_{5} \times S^{5}$ type IIB supergravity [14]. The authors of [14] had to impose both (5.1) and (5.3) since they evaluated explicitly only the Helmholtz free energy density and the temperature, while constraining the entropy density and the energy density from the consistency of the thermodynamics ${ }^{14}$. Though there are few explicit checks of the $\alpha^{\prime}$-corrected black hole thermodynamics, we would like to stress that validity of (5.1)-(5.4) for $\alpha^{\prime}$-corrected black holes studied here are guaranteed by the gauge theory-string theory correspondence [1]. The reason for this is that the $\alpha^{\prime}$-corrected black holes studied here are dual to supersymmetric $\mathcal{N}=4$ $S U\left(n_{c}\right)$ Yang-Mills theory at finite temperature, certain $U(1)_{R}$ chemical potentials and at finite 't Hooft coupling, corresponding to finite $\alpha$ ' corrections.

In this paper we use holographic renormalization (to leading order in $\alpha^{\prime}$ ) to compute the free energy density and the energy density of a given black hole. Furthermore, we evaluate the $\alpha^{\prime}$-corrected temperature of a black hole as inverse periodicity of the Euclidean time direction, requiring the absence of conical singularities. In the case of neutral black holes we use (5.1) to compute the entropy density. The first law of thermodynamics (5.3) then provides a nontrivial consistency check on our analysis. For the charged black holes we can use (5.2) to evaluate the entropy density. The remaining thermodynamic characteristics, i.e., the chemical potential and the physical

\footnotetext{
${ }^{13}$ For a sample see $[9,11,12,29,35,36]$.

${ }^{14}$ Recently is was demonstrated in [37] that the entropy density computed in [14] agrees with the Wald formula [38] for the black hole entropy in higher derivative gravity. Wald formula was tested as well in other models of higher curvature gravity [39-43].
} 
charge, are then evaluated imposing the first law of thermodynamics (5.4). In a similar way one can study thermodynamics of $\alpha^{\prime}$-corrected single-charge black holes, but our approach can not be applied to black holes with two or more different $U(1)$ charges.

It is convenient to introduce "hatted" thermodynamic potentials as

$$
\Omega \equiv \frac{n_{c}^{2}}{8 \pi^{2}} \hat{\Omega}, \quad F \equiv \frac{n_{c}^{2}}{8 \pi^{2}} \hat{F}, \quad E \equiv \frac{n_{c}^{2}}{8 \pi^{2}} \hat{E}, \quad S \equiv \frac{n_{c}^{2}}{8 \pi^{2}} \hat{S}, \quad \mu \equiv \frac{n_{c}^{2}}{8 \pi^{2}} \hat{\mu}_{q} .
$$

Consider first computation of the Gibbs free energy density ${ }^{15}$. The free energy is defined as

$$
\frac{\operatorname{vol}\left(S^{3}\right)}{\kappa^{3 / 2}} \Omega=T I_{E},
$$

where $I_{E}$ is the Euclidean action of the "on-shell" gravitational background. The latter is divergent and thus should be regularized and holographically renormalized. Define regularized Euclidean bulk effective action $S_{E, \text { eff }}^{\rho}$ by cutting off the radial coordinate integration

$$
\int_{r_{+}}^{\infty} d r \rightarrow \int_{r_{+}}^{\rho} d r
$$

in (3.8) and (3.9) at some UV cutoff $r=\rho \gg r_{+}$. Holographically renormalized action $I_{E}^{\rho}$ is then obtained by supplementing $S_{E, \text { eff }}^{\rho}$ with the Gibbons-Hawking and the boundary counterterms

$$
I_{E}^{\rho}=S_{E, \text { eff }}^{\rho}+I_{G H}+I_{\text {counterterms }}
$$

so that no divergences arise in $\rho \rightarrow \infty$ limit,

$$
I_{E}=\lim _{\rho \rightarrow \infty} I_{E}^{\rho}
$$

The Gibbons-Hawking term is most easy to evaluate in ten dimensions. It is given by the integral over the nine-dimensional boundary $\partial \mathcal{M}_{10}$ located at $r=\rho$

$$
I_{G H}=-\frac{1}{8 \pi G_{10}} \int_{\partial \mathcal{M}_{10}, r=\rho} d^{9} \xi \sqrt{h_{E}} \nabla_{\mu} n^{\mu},
$$

where $h_{E}$ is induced metric on $\partial \mathcal{M}_{10}$ and $n^{\mu}=D_{3}^{-1} \delta_{r}^{\mu}$ is a unit outward normal to this boundary. For the boundary counterterms we would like to use results of $[11,12]$. For this reason we express the counterterm action $I_{\text {counterterm }}$ as

$$
I_{\text {counterterms }}=\frac{1}{4 \pi G_{5}} \int_{\partial \mathcal{M}_{5}, r=\rho} d^{4} \xi\left(\alpha_{1} \sqrt{H_{E}}+\alpha_{2} R_{4} \sqrt{H_{E}}\right),
$$

\footnotetext{
${ }^{15}$ The Gibbs free energy reduces to Helmholtz free energy in the absence of the chemical potentials.
} 
where $\partial \mathcal{M}_{5}$ is a Kaluza-Klein reduction of $\partial \mathcal{M}_{10}$, the five-dimensional Newton's constant is $G_{5}=\frac{G_{10}}{\pi^{3}}$, and $R_{4}$ is a Ricci scalar evaluated on the metric $H_{E, \mu \nu}$ which is induced on the $\partial \mathcal{M}_{5}$ boundary from the five-dimensional Kaluza-Klein reduced metric

$$
G_{E, \mu \nu} d \xi^{\mu} d \xi^{\nu}=D_{4}^{10 / 3}\left(D_{1}^{2} d t^{2}+D_{2}^{2} \frac{1}{\kappa}\left(S^{3}\right)^{2}+D_{3}^{2} d r^{2}\right)
$$

In the supergravity approximation (see [11] where the same notations have been used)

$$
\alpha_{1}=\frac{3}{2}, \quad \alpha_{2}=\frac{1}{8} .
$$

Though in principle $\alpha_{i}$ in (5.13) might receive $\alpha^{\prime}$-corrections, for the class of black holes discussed here, this does not happen. The reason is that $\alpha^{\prime}$-corrections to the charged black hole metric vanish sufficiently fast at asymptotic infinity and does not affect the divergences of $S_{E, \text { eff }}^{\rho}$ as $\rho \rightarrow \infty$.

Since each contribution of $I_{E}^{\rho}$ is evaluated with the $\alpha^{\prime}$-corrected on-shell metric (3.14), we can naturally split (to leading order in $\gamma(3.2)$ )

$$
\begin{aligned}
& I_{E}^{\rho} \equiv I_{0, E}^{\rho}+\gamma \delta I_{E} \\
& =\left(S_{0, E, \text { eff }}^{\rho}+I_{0, G H}+I_{0, \text { counterterms }}\right)+\gamma\left(\delta I_{S U G R A}+\delta I_{W}+\delta I_{G H}+\delta I_{\text {counterterms }}\right),
\end{aligned}
$$

where the subscript ${ }_{0}$ indicates purely supergravity contributions, while $\delta \cdots$ denotes $\alpha^{\prime}$-corrections. Notice that

$$
\frac{T}{\operatorname{vol}\left(S^{3}\right) / \kappa} \lim _{r \rightarrow \infty} I_{0, E}^{\rho}
$$

gives precisely the Gibbs free energy density in (2.10).

The $\delta I_{S U G R A}$ contribution comes the supergravity part (3.8) of the effective action (3.7). Due to the diffeomorphism invariance, much like $S_{0, E, \text { eff }}, \delta I_{S U G R A}$ can be written as a total derivative involving the deformations $\{A, B, \nu, a\}$ in (3.14), and thus receives contributions only from the regularization $r=\rho$ boundary and the horizon $r=r_{+}$. 
Explicitly,

$$
\begin{aligned}
\delta I_{S U G R A}= & \frac{\operatorname{vol}\left(S^{3}\right)}{T \kappa^{3 / 2}} \frac{\pi^{3}}{16 \pi G_{10}}\left(\left(\left(4 Q \mu+4 \kappa Q^{2}\right)+(-4 \mu-8 \kappa Q) x+4 \kappa x^{2}+4 x^{3}\right) B^{\prime}\right. \\
& -\frac{2}{3 x}\left(\left(-24 Q \mu-24 \kappa Q^{2}\right) x+(48 \kappa Q+24 \mu) x^{2}-24 \kappa x^{3}-24 x^{4}\right) A^{\prime} \\
& -\frac{2}{x}\left(\left(Q \mu+\kappa Q^{2}\right)+(\mu+2 \kappa Q) x-3 \kappa x^{2}-5 x^{3}\right) B \\
& -\frac{2}{x}\left(\left(Q \mu+\kappa Q^{2}\right)+(-4 \kappa Q-2 \mu) x+3 \kappa x^{2}+4 x^{3}\right) A \\
& -\frac{20}{3}\left(\left(Q \mu+\kappa Q^{2}\right)+(-\mu-2 \kappa Q) x+\kappa x^{2}+x^{3}\right) \nu^{\prime} \\
& \left.+\frac{2 Q(\mu+\kappa Q)}{x} a\right)\left.\right|_{x=x_{+}} ^{x=\rho^{2}+Q},
\end{aligned}
$$

where we used the fact that Euclidean time direction is periodic with periodicity $\frac{1}{T}$. Notice that the coefficient in front of $\nu^{\prime}$ in (5.15) vanishes ${ }^{16}$ for $x=x_{+}$, thus even though for $\kappa \neq 0$ neutral black holes $(Q=0)$ we can not find the analytic solution for $\nu(x)$, we can explicitly compute the $\alpha^{\prime}$ corrections contributed by (5.15). For the charged black holes we need to know the value of the gauge potential $a$ at the horizon, which through $\mathcal{C}_{a}$ (4.25) ( or (4.38) ) requires the knowledge of $\nu(x)$. In fact, above is the only place where the $\alpha^{\prime}$-corrected thermodynamics of charged black holes in asymptotic $A d S_{5} \times S^{5}$ geometry is sensitive to the five-sphere warp factor $\nu$.

The $\delta I_{W}$ is most easy to evaluate as a (convergent) bulk integral, much like it was done in [14]

$$
\delta I_{W}=-\frac{v o l\left(S^{3}\right)}{T \kappa^{3 / 2}} \frac{\pi^{3}}{16 \pi G_{10}} \int_{r_{+}}^{\infty} d r D_{1}^{(0)}\left[D_{2}^{(0)}\right]^{3} D_{3}^{(0)}\left[D_{5}^{(0)}\right]^{5} W\left[D_{i}^{0}, D a^{(0)}\right]
$$

Notice that the integrand in (5.16) is evaluated with the $a^{\prime}$-uncorrected metric (3.13).

Finally, $\delta I_{G H}$ and $\delta I_{\text {counterterms }}$ are simply the contributions $\propto \gamma$ coming from (5.10) and (5.11) (with (5.13)) when evaluated with $\alpha^{\prime}$-corrected metric (3.14).

In addition to the Gibbs free energy density we need to evaluate the energy density, E. We find $^{17}$

$$
\frac{\operatorname{vol}\left(S^{3}\right)}{\kappa^{3 / 2}} E=\int_{\Sigma} d^{3} \xi \sqrt{\sigma} N_{\Sigma} \epsilon
$$

\footnotetext{
${ }^{16}$ This term does not contribute when evaluated at the boundary $x=\rho^{2}+Q$ either as $\nu(x)$ vanishes fast enough, see (4.12).

${ }^{17}$ We follow here the presentation of [11].
} 
where $\Sigma \equiv S^{3}$ is a spacelike hypersurface in $\partial \mathcal{M}_{5}$ with a timelike unit normal $u^{\mu}, N_{\Sigma}$ is the norm of the timelike Killing vector in (5.12), $\sigma$ is the determinant of the induced metric on $\Sigma$, and $\epsilon$ is the proper energy density

$$
\epsilon=u^{\mu} u^{\nu} T_{\mu \nu}
$$

The quasilocal stress tensor $T_{\mu \nu}$ for our background is obtained from the variation of the Kaluza-Klein reduced Gibbons-Hawking term (5.10) and the boundary counterterms $(5.11)^{18}$ with respect to the boundary metric $\delta H_{\mu \nu}$

$$
T^{\mu \nu}=\frac{2}{\sqrt{-H}} \frac{\delta}{\delta H_{\mu \nu}}\left[I_{G H}+I_{\text {counterterms }}\right] \text {. }
$$

Explicit computation yields

$$
T^{\mu \nu}=\frac{1}{8 \pi G_{5}}\left[-\Theta^{\mu \nu}+\Theta H^{\mu \nu}-2 \alpha_{1} H^{\mu \nu}+4 \alpha_{2}\left(R_{4}^{\mu \nu}-\frac{1}{2} R_{4} H^{\mu \nu}\right)\right],
$$

where

$$
\Theta^{\mu \nu}=\frac{1}{2}\left(\nabla^{\mu} n^{\nu}+\nabla^{\nu} n^{\mu}\right), \quad \Theta=\operatorname{Tr} \Theta^{\mu \nu}
$$

\subsection{Thermodynamics of neutral black holes}

We use the framework detailed in Section 5.1 to study the thermodynamics of $\alpha^{\prime}$ corrected neutral black holes in asymptotic $A d S_{5} \times S^{5}$ type IIB supergravity with the spatial horizon curvature $\kappa=\{0,1\}$. The relevant formulas for the $\alpha^{\prime}$-corrected geometry are given in Section 4.1. The only other information we need is the value of the quartic Weyl invariant $W(3.3)$ evaluated in the supergravity approximation. We find

$$
W=\frac{180 \mu^{4}}{r^{16}}
$$

Recalling that the horizon location is given by the largest positive root of (2.7),

$$
0=x_{+}^{2}+x_{+} \kappa-\mu
$$

explicit evaluation of various contribution in (5.14) yields

$$
\delta I_{\text {SUGRA }}=\frac{\operatorname{vol}\left(S^{3}\right)}{T \kappa^{3 / 2}} \frac{\pi^{3}}{16 \pi G_{10}} \times\left\{-\frac{15 \mu^{3}}{7 x_{+}^{6}}\left(31 \mu-24 \kappa x_{+}\right)\right\},
$$

\footnotetext{
${ }^{18}$ There is no contribution from the bulk effective action (3.7).
} 


$$
\begin{gathered}
\delta I_{W}=\frac{\operatorname{vol}\left(S^{3}\right)}{T \kappa^{3 / 2}} \frac{\pi^{3}}{16 \pi G_{10}} \times\left\{-\frac{15 \mu^{4}}{x_{+}^{6}}\right\}, \\
\delta I_{G H}=\frac{\operatorname{vol}\left(S^{3}\right)}{T \kappa^{3 / 2}} \frac{\pi^{3}}{16 \pi G_{10}} \times\left\{\frac{20 \mu^{3}}{7 x_{+}^{6}}\left(57 \mu-32 \kappa x_{+}\right)\right\}, \\
\delta I_{\text {counteterms }}=\frac{\operatorname{vol}\left(S^{3}\right)}{T \kappa^{3 / 2}} \frac{\pi^{3}}{16 \pi G_{10}} \times\left\{-\frac{15 \mu^{3}}{7 x_{+}^{6}}\left(57 \mu-32 \kappa x_{+}\right)\right\},
\end{gathered}
$$

so that for the Helmholtz free energy density we find

$$
\hat{F}=-\mu+\frac{3}{4} \kappa^{2}+2 \kappa x_{+}+\gamma \times\left\{-\frac{5 \mu^{3}}{7 x_{+}^{6}}\left(57 \mu-40 \kappa x_{+}\right)\right\} .
$$

For the energy density $E(5.17)$ we find

$$
\hat{E}=3 \mu+\frac{3}{4} \kappa^{2}+\gamma \times\left\{\frac{3}{28 x_{+}^{2}}\left(\left(500 \kappa^{3} x_{+}+500 \kappa^{4}\right)+\left(2140 \kappa^{2}+1640 \kappa x_{+}\right) \mu+1140 \mu^{2}\right)\right\} .
$$

Finally, the Hawking temperature is

$$
\pi T=\frac{\mu}{2 x_{+}^{3 / 2}}+\frac{\sqrt{x_{+}}}{2}+\gamma \times\left\{\frac{5}{7 x_{+}^{7 / 2}}\left(-\kappa^{4}-\kappa^{3} x_{+}+\left(8 \kappa x_{+}+7 \kappa^{2}\right) \mu+9 \mu^{2}\right)\right\} .
$$

Using the basic thermodynamic relation (5.1) we find the entropy density

$$
\hat{S}=4 \pi x_{+}^{3 / 2}+\gamma \times\left\{\frac{960 \pi}{7 x_{+}^{3 / 2}}\left(\kappa^{2} x_{+}+\kappa^{3}+\left(x_{+}+2 \kappa\right) \mu\right)\right\} .
$$

It is straightforward to verify that for arbitrary spatial horizon curvature $\kappa$ and to leading order in $\gamma$, the first law of thermodynamics (5.3) is automatically satisfied.

We have now all the necessary information to compute the $\alpha^{\prime}$ corrections to the Hawking-Page phase transition temperature. Since global $A d S_{5} \times S^{5}$ geometry does not receive $\alpha^{\prime}$ corrections to leading order (see Section 4.1), the transition temperature $T_{H P}$ is determined from the condition

$$
\hat{F}(\mu)=\hat{E}(\mu=0, \gamma=0) \quad \Rightarrow \quad \pi T_{H P}=\frac{3}{2} \kappa^{1 / 2}\{1-40 \gamma\},
$$

where $\hat{F}$ and $\hat{E}$ are given in (5.28) and (5.29) correspondingly.

\subsection{Thermodynamics of charge black branes at $\mu_{q} \ll T$}

We use the framework detailed in Section 5.1 to study the thermodynamics of $\alpha^{\prime}$ corrected charged black holes in asymptotic $A d S_{5} \times S^{5}$ type IIB supergravity with the 
spatial horizon curvature $\kappa=0$ and in the high temperature regime, i.e., $T \gg \mu_{q}$. The relevant formulas for the $\alpha^{\prime}$-corrected geometry are given in Section 4.3 (or Appendix C). The only other information we need is the value of the quartic Weyl invariant $W$ (3.3) evaluated in the supergravity approximation. We find ${ }^{19}$

$$
W=\frac{180 \mu^{4}}{x^{8}}-\frac{240 \mu^{3} Q}{x^{9}}\left(4 \mu+x^{2}\right) .
$$

Recalling that the horizon location is given by (see (C.1)),

$$
x_{+}=\sqrt{\mu}-\frac{1}{2} Q
$$

explicit evaluation of various contribution in (5.14) yields

$$
\begin{gathered}
\delta I_{\text {SUGRA }}=\frac{\operatorname{vol}\left(S^{3}\right)}{T \kappa^{3 / 2}} \frac{\pi^{3}}{16 \pi G_{10}} \times\left\{-\frac{465}{7} \mu+Q\left(\frac{4245}{7}+\frac{3853}{18} \sqrt{\mu}\right)\right\}, \\
\delta I_{W}=\frac{\operatorname{vol}\left(S^{3}\right)}{T \kappa^{3 / 2}} \frac{\pi^{3}}{16 \pi G_{10}} \times\left\{-15 \mu+\frac{333}{7} \sqrt{\mu} Q\right\} \\
\delta I_{G H}=\frac{\operatorname{vol}\left(S^{3}\right)}{T \kappa^{3 / 2}} \frac{\pi^{3}}{16 \pi G_{10}} \times\left\{\frac{1140}{7} \mu-\frac{32749}{63} \sqrt{\mu} Q\right\} \\
\delta I_{\text {counteterms }}=\frac{\operatorname{vol}\left(S^{3}\right)}{T \kappa^{3 / 2}} \frac{\pi^{3}}{16 \pi G_{10}} \times\left\{-\frac{855}{7} \mu+\frac{32749}{84} \sqrt{\mu} Q\right\},
\end{gathered}
$$

so that for the Gibbs free energy density we find

$$
\hat{\Omega}=-\mu+\gamma \times\left\{-\frac{285}{7} \mu+\left(\frac{33181}{252} \sqrt{\mu}+\frac{4245}{7}\right) Q\right\} .
$$

For the energy density $E(5.17)$ we find

$$
\hat{E}=3 \mu+\gamma \times\left\{\frac{855}{7} \mu-\frac{32749}{84} \sqrt{\mu} Q\right\} .
$$

Finally, the Hawking temperature is

$$
\pi T=\mu^{1 / 4}-\frac{3}{4 \mu^{1 / 4}} Q+\gamma \times\left\{\frac{45}{7} \mu^{1 / 4}+\frac{2243}{1008 \mu^{1 / 4}} Q\right\} .
$$

As explained in Section 5.1, we use the basic thermodynamic relation (5.2) and the first law of thermodynamics (5.4) to determine the remaining thermodynamic potentials, i.e., the entropy density $\hat{S}$, the chemical potential $\hat{\mu}_{q}$ and the physical charge

\footnotetext{
${ }^{19}$ All expressions in this section are to leading order in $Q$.
} 
density $\tilde{Q}$. Specifically, we parameterize the chemical potential $\hat{\mu}_{q}$ and the physical charge $\tilde{Q}$ (to leading order in $\gamma$ and to leading order in $Q$ ) as

$$
\hat{\mu}_{q}=2 Q^{1 / 2}\left(1+\gamma \delta \mu_{q}(\mu)\right), \quad \tilde{Q}=Q^{1 / 2}(\sqrt{\mu}+\gamma \delta q(\mu)) .
$$

Next, we evaluate the entropy density from (5.2), and impose the first law of thermodynamics (5.4)

$$
\begin{aligned}
0= & d \hat{\Omega}+\hat{S} d T+3 \tilde{Q} d \hat{\mu}_{q}=\gamma \times\left\{\frac{264}{7} \mu^{1 / 2}+\frac{4245}{7}+3 \delta q(\mu)+3 \sqrt{\mu} \delta \mu_{q}(\mu)\right\} d(Q) \\
& +\gamma \times\left\{-\frac{4245}{28 \mu}-\frac{123}{7 \sqrt{\mu}}-\frac{3}{2 \mu} \delta q(\mu)+6 \sqrt{\mu} \frac{d}{d \mu} \delta \mu_{q}(\mu)-\frac{3}{2 \sqrt{\mu}} \delta \mu_{q}(\mu)\right\} Q d(\mu) .
\end{aligned}
$$

From (5.43) we find

$$
\delta \mu_{q}(\mu)=-\frac{3}{14} \ln \frac{\mu}{\pi^{4} \epsilon^{4}}+\frac{1415}{28 \sqrt{\mu}}-\frac{89}{7}, \quad \delta q(\mu)=-\frac{7075}{28}+\frac{1}{7} \sqrt{\mu}+\frac{3}{14} \sqrt{\mu} \ln \frac{\mu}{\pi^{4} \epsilon^{4}} .
$$

where $\epsilon$ is an arbitrary energy scale, arising as an integration constant in solving (5.43). Though $\hat{\mu}_{q}$ and $\tilde{Q}$ separately depend on $\epsilon$, their product $\hat{\mu}_{q} \tilde{Q}$, and thus via (5.2) the entropy density $\hat{S}$, is $\epsilon$ independent. It would be interesting to repeat computation of [37] and compare the entropy density obtained here from the thermodynamics with the Wald formula [38]. We comment on the physical meaning of the energy scale $\epsilon$ in the conclusion section.

In the rest of this section we express all thermodynamic properties of the $\alpha^{\prime}$ corrected charged black holes discussed here in terms of the temperature $T$ and the physical charge chemical potential $\mu_{q}$. We find ${ }^{20}$

$$
\begin{aligned}
\hat{\Omega}= & -\pi^{4} T^{4}\left\{\left(1+15 \times \gamma+\mathcal{O}\left(\gamma^{2}\right)\right)\right. \\
& +\frac{3 \mu_{q}^{2}}{4 \pi^{2} T^{2}}\left(1-\gamma \times\left(\frac{4245}{14 \pi^{2} T^{2}}+\frac{12}{7} \ln \frac{\epsilon}{T}\right)+\mathcal{O}\left(\gamma^{2}\right)\right) \\
& \left.+\mathcal{O}\left(\frac{\mu_{q}^{4}}{T^{4}}\right)(1+\mathcal{O}(\gamma))\right\},
\end{aligned}
$$

\footnotetext{
${ }^{20}$ Correct dimensionality can be restored with $L$, with was set to unity.
} 


$$
\begin{aligned}
\hat{E}= & 3 \pi^{4} T^{4}\left\{\left(1+15 \times \gamma+\mathcal{O}\left(\gamma^{2}\right)\right)\right. \\
& +\frac{3 \mu_{q}^{2}}{4 \pi^{2} T^{2}}\left(1+\gamma \times\left(\frac{4}{7}-\frac{1415}{14 \pi^{2} T^{2}}-\frac{12}{7} \ln \frac{\epsilon}{T}\right)+\mathcal{O}\left(\gamma^{2}\right)\right) \\
+ & \left.\mathcal{O}\left(\frac{\mu_{q}^{4}}{T^{4}}\right)(1+\mathcal{O}(\gamma))\right\} \\
\hat{S}= & 3 \pi^{4} T^{3}\left\{\left(1+15 \times \gamma+\mathcal{O}\left(\gamma^{2}\right)\right)\right. \\
& +\frac{3 \mu_{q}^{2}}{8 \pi^{2} T^{2}}\left(1+\gamma \times\left(\frac{6}{7}-\frac{12}{7} \ln \frac{\epsilon}{T}\right)+\mathcal{O}\left(\gamma^{2}\right)\right) \\
& \left.+\mathcal{O}\left(\frac{\mu_{q}^{4}}{T^{4}}\right)(1+\mathcal{O}(\gamma))\right\}, \\
\tilde{Q}=\frac{1}{2} \mu_{q} \pi^{2} T^{2}\{(1- & \left.\left.\gamma\left(\frac{4245}{14 \pi^{2} T^{2}}+\frac{12}{7} \ln \frac{\epsilon}{T}\right)+\mathcal{O}\left(\gamma^{2}\right)\right)+\mathcal{O}\left(\frac{\mu_{q}^{2}}{T^{2}}\right)(1+\mathcal{O}(\gamma))\right\} .
\end{aligned}
$$

Notice that in the absence of chemical potential, $\mu_{q}=0$, we reproduce results of $[14,15]$.

\section{Conclusion}

In this paper we studied leading $a^{\prime}$ corrections to charged near-extremal black holes in asymptotic $A d S_{5} \times S^{5}$ type IIB supergravity. We used $\alpha^{\prime}$-corrected holographic renormalization to evaluate the free energy density and the ADM mass density of the black holes under the assumption that the five-form does not receive $\alpha^{\prime}$ corrections. We extracted the remaining thermodynamic properties imposing the basic thermodynamic relations ((5.1) or (5.2)) along with the first law of thermodynamics.

In the case of neutral black holes with spatial horizon curvature $\kappa$ we showed that given the Helmholtz free energy and the energy density, the entropy density obtained from the basic thermodynamic relation (5.1) automatically satisfies the first law of thermodynamics. We evaluated leading $\alpha^{\prime}$ corrections to the Hawking-Page phase transition temperature. Hawking-Page phase transition is a holographic dual [6] to a confinement-deconfinement phase transition in $\mathcal{N}=4 S U\left(n_{c}\right)$ SYM compactified on $S^{3}$, which was extensively studied recently at weak t' Hooft coupling in $[44,45]$.

Our computational approach allows to study either black holes with a single nonzero charge under the $\left[U(1)_{R}\right]^{3}$ symmetry or black holes with all identical $U(1)_{R}$ symmetry charges. In this paper we discussed technically less complicated case of iden- 
tical charges ${ }^{21}$. Apart from the $S^{5}$ sphere warp factor, we analytically solved for the $\alpha^{\prime}$-corrected nonextremal black hole geometry with the spatial horizon curvature $\kappa=\{0,1\}$. Unlike the neutral black holes, the knowledge of the latter warp factor is vital for understanding $\alpha^{\prime}$ corrected thermodynamic properties of charged black holes. We solved for the $S^{5}$ warp factor of the $\kappa=0$ charged black hole analytically in the high temperature regime, when the temperature is much larger than the chemical potential. Rather interestingly, we found that in the high temperature regime, the Gibbs free energy density of these black holes receives correction (5.45)

$$
\delta \Omega \propto-\left(\alpha^{\prime}\right)^{3} T^{2} \mu_{q}^{2} \ln \frac{T}{\epsilon}
$$

where $\epsilon$ is an arbitrary scale. From the dual gauge theory perspective it appears that the Gibbs free energy of the $\mathcal{N}=4 \mathrm{SYM}$ at finite temperature, chemical potential and a finite (but large) 't Hooft coupling depends on arbitrary energy scale $\epsilon$. How is it possible?

To understand this puzzle, consider four dimensional, non-supersymmetric QCD with quarks, at finite temperature. To two-loop order using hard-thermal-loop perturbation theory the Helmholtz free energy density (or minus the pressure) is given by $[46]$

$F_{Q C D}=-\frac{8 \pi^{2}}{45} T^{4}\left[\mathcal{F}_{0}+\mathcal{F}_{2} \frac{\alpha_{s}}{\pi}+\mathcal{F}_{3}\left(\frac{\alpha_{s}}{\pi}\right)^{3 / 2}+\mathcal{F}_{4}\left(\frac{\alpha_{s}}{\pi}\right)^{2}+\mathcal{F}_{5}\left(\frac{\alpha_{s}}{\pi}\right)^{5 / 2}+\mathcal{O}\left(\alpha_{s}^{3} \ln \alpha_{s}\right)\right]$

where $\mathcal{F}_{4}$ and $\mathcal{F}_{5}$ contain an explicit $\ln \frac{\epsilon}{T}$ dependence on the renormalization scale $\epsilon$. There is no contradiction here, because taking into account the running coupling constant $\alpha_{s}=\alpha_{s}(\epsilon)$ one finds ${ }^{22}$ that

$$
\epsilon \frac{d}{d \epsilon} F_{Q C D}=\mathcal{O}\left(\alpha_{s}^{3} \ln \alpha_{s}\right)
$$

Turning things around, appearance of the $\ln \frac{\epsilon}{T}$ signals the $\epsilon$-dependence ( the familiar perturbative running) of the gauge coupling $\alpha_{s}$. Thus, we are tempted to conjecture that appearance of an arbitrary energy scale $\epsilon$ in (6.1) signals the scale dependence, "running" of the inverse 't Hooft coupling $\lambda^{-1}=1 /\left(g_{Y M}^{2} n_{c}\right) \propto \gamma^{3 / 2}=\gamma^{3 / 2}(\epsilon)$ in $\mathcal{N}=4$

\footnotetext{
${ }^{21}$ The thermodynamics of single-charge nonextremal black holes in asymptotic $A d S_{5} \times S^{5}$ type IIB supergravity will be discussed elsewhere.

${ }^{22}$ Renormalization group resummation of the free energy of hot QCD was discussed in [47].
} 
SYM. If so, requiring that $\hat{\Omega}$ in (5.45) is a renormalization group (RG) invariant to order computed, i.e.,

$$
\epsilon \frac{d}{d \epsilon} \hat{\Omega}=0+\mathcal{O}\left(\gamma^{2}\right)+\mathcal{O}\left(\frac{\mu_{q}^{4}}{T^{4}}\right)(1+\mathcal{O}(\gamma)),
$$

we find

$$
\epsilon \frac{d}{d \epsilon} \gamma=\frac{3 \mu_{q}^{2}}{35 \pi^{2} T^{2}} \gamma
$$

The RG running (6.5) is to be understood as running of the effective $\mathcal{N}=4$ coupling at energy scales below $T$, which is the highest scale in the problem. We do not expect running at energies much higher than the temperature scale. In this respect the situation is similar to a mass deformation of the $\mathcal{N}=4$ SYM: above the mass scale, the SYM coupling is constant, while the effective Wilsonian coupling runs below the mass scale since some (or all) of the scalars and fermions of the $\mathcal{N}=4$ supersymmetric Yang-Mills are integrated out.

Finally, if there is running of the effective $\mathcal{N}=4$ SYM coupling at strong coupling (and nonzero chemical potential) it is natural to conjecture that SYM coupling would also run at weak 't Hooft coupling and temperatures much larger than the chemical potential. The structure of the perturbative (in the 't Hooft coupling $\lambda$ ) corrections to a hot QCD pressure [46] and that of the $\mathcal{N}=4$ gauge theory plasma [48-50] is similar to order $\lambda^{3 / 2}$. Unfortunately, there is no computation in thermal $\mathcal{N}=4$ available to order $\lambda^{2}$, which is the first order in perturbation theory where $\ln \frac{\epsilon}{T}$ correction appears in QCD (6.2). Actually, we expect that the $\mathcal{N}=4$ gauge coupling will run only when the chemical potential is nonzero. But again, currently, the computations with nonzero chemical potential are available only to order $\lambda^{3 / 2}[51]$.

\section{Acknowledgments}

I would like to thanks Philip Argyres, Stan Deakin, Jaume Gomis, Gerry Mckeon, Rob Myers and Volodya Miransky for valuable discussions. I would like to thank Banff International Research Station, Michigan Center for Theoretical Physics and University of Cincinnati for hospitality where part of this work was done. Research at Perimeter Institute is supported in part by funds from NSERC of Canada. I gratefully acknowledge support by NSERC Discovery grant. 


\section{Appendix}

\section{A Data for charged $\kappa=1$ black holes}

Inhomogeneous parts of equations (4.17)-(4.21):

$$
\begin{aligned}
J_{A}= & -\frac{1}{12 x^{11}}\left(-160 Q(Q+\mu)(2 Q+\mu) x^{4}+\left(-23040 Q^{3}+912 \mu^{2} Q^{2}+912 Q^{4}\right.\right. \\
& \left.-34560 \mu Q^{2}-4320 \mu^{3}-20160 \mu^{2} Q+1824 Q^{3} \mu\right) x^{3}+6 Q(Q+\mu)\left(5359 \mu^{2}\right. \\
& \left.\left.+18440 Q \mu+18440 Q^{2}\right) x^{2}-84196 Q^{2}(Q+\mu)^{2}(2 Q+\mu) x+82707 Q^{3}(Q+\mu)^{3}\right) \\
J_{B}= & 8 x^{11}\left(x^{2}-2 x Q+Q^{2}+x^{3}-\mu x+Q \mu\right) \\
& \left.-2304 Q^{4}-84672 \mu Q^{2}-56448 Q^{3}-11520 \mu^{3}-51264 \mu^{2} Q-2304 \mu^{2} Q^{2}\right) x^{6} \\
& +\left(553536 Q^{3} \mu+360304 \mu^{2} Q^{2}-50560 \mu^{2} Q-56320 Q^{3}+83536 \mu^{3} Q-11200 \mu^{3}\right. \\
& \left.-84480 \mu Q^{2}+276768 Q^{4}\right) x^{5}+\left(-428544 Q^{5}+11160 \mu^{4}-1071360 \mu Q^{4}\right. \\
& +155468 \mu^{3} Q-214272 \mu^{3} Q^{2}+389600 Q^{4}+779200 Q^{3} \mu+545068 \mu^{2} Q^{2} \\
& \left.-857088 \mu^{2} Q^{3}\right) x^{4}+8 Q(Q+\mu)\left(26678 Q^{4}+53356 Q^{3} \mu-129602 Q^{3}+26678 \mu^{2} Q^{2}\right. \\
& \left.-194403 \mu Q^{2}-88139 \mu^{2} Q-11669 \mu^{3}\right) x^{3}+2 Q^{2}(Q+\mu)^{2}\left(147484 \mu^{2}+670389 Q \mu\right. \\
& \left.\left.+670389 Q^{2}\right) x^{2}-423932 Q^{3}(Q+\mu)^{3}(2 Q+\mu) x+210625 Q^{4}(Q+\mu)^{4}\right)
\end{aligned}
$$$$
J_{\nu}=\frac{1}{320 x^{11}\left(x^{2}-2 x Q+Q^{2}+x^{3}-\mu x+Q \mu\right)}\left(47488 Q(Q+\mu)(2 Q+\mu) x^{7}\right.
$$$$
-64 Q(Q+\mu)\left(-1624 \mu+2511 Q \mu-3248 Q+2511 Q^{2}\right) x^{6}+\left(-982784 \mu^{2} Q^{2}\right.
$$$$
-850240 Q^{4}-15360 \mu^{2} Q-15360 \mu^{3}+30720 Q^{3}-1700480 Q^{3} \mu+46080 \mu Q^{2}
$$$$
\left.-132544 \mu^{3} Q\right) x^{5}+\left(31320 \mu^{4}-63360 Q^{3} \mu+561344 \mu^{3} Q^{2}+2806720 \mu Q^{4}\right.
$$$$
\left.+1122688 Q^{5}-31680 Q^{4}+2245376 \mu^{2} Q^{3}+169728 \mu^{3} Q+138048 \mu^{2} Q^{2}\right) x^{4}
$$$$
-16 Q(Q+\mu)\left(30081 Q^{4}+60162 Q^{3} \mu+17464 Q^{3}+30081 \mu^{2} Q^{2}+26196 \mu Q^{2}\right.
$$$$
\left.+28376 \mu^{2} Q+9822 \mu^{3}\right) x^{3}+4 Q^{2}(Q+\mu)^{2}\left(73187 \mu^{2}+188384 Q \mu+188384 Q^{2}\right) x^{2}
$$$$
\left.-349232 Q^{3}(Q+\mu)^{3}(2 Q+\mu) x+225375 Q^{4}(Q+\mu)^{4}\right)
$$ 


$$
\begin{aligned}
J_{a}= & \frac{1}{2 x^{11}}\left(-576 Q(Q+\mu) x^{6}+\left(3840 \mu^{2}+9984 Q^{2}+9984 Q \mu\right) x^{5}+\left(-33440 Q^{3}\right.\right. \\
& \left.+13440 Q^{2}+13440 Q \mu-50160 \mu Q^{2}+4800 \mu^{2}-16720 \mu^{2} Q\right) x^{4}+\left(25464 Q^{4}\right. \\
& \left.-104688 \mu Q^{2}-44976 \mu^{2} Q+25464 \mu^{2} Q^{2}-5040 \mu^{3}-69792 Q^{3}+50928 Q^{3} \mu\right) x^{3} \\
& +14 Q(Q+\mu)\left(1909 \mu^{2}+9372 Q \mu+9372 Q^{2}\right) x^{2}-53200 Q^{2}(2 Q+\mu)(Q+\mu)^{2} x \\
+ & \left.31473 Q^{3}(Q+\mu)^{3}\right) \\
J_{\text {const }}= & -\frac{1}{24 x^{10}}\left(1696 Q(Q+\mu)(2 Q+\mu) x^{7}-128 Q(Q+\mu)\left(31 Q \mu-22 \mu+31 Q^{2}\right.\right. \\
& -44 Q) x^{6}+\left(-31352 \mu^{2} Q^{2}-11520 \mu Q^{2}-7680 Q^{3}-51392 Q^{3} \mu-5656 \mu^{3} Q\right. \\
& \left.-7680 \mu^{2} Q-25696 Q^{4}-1920 \mu^{3}\right) x^{5}+\left(81192 \mu^{2} Q^{2}+27816 \mu^{3} Q+101600 \mu Q^{4}\right. \\
& \left.+81280 \mu^{2} Q^{3}+106752 Q^{3} \mu+3240 \mu^{4}+20320 \mu^{3} Q^{2}+53376 Q^{4}+40640 Q^{5}\right) x^{4} \\
& -4 Q(Q+\mu)\left(5211 Q^{4}+10422 Q^{3} \mu+37072 Q^{3}+5211 \mu^{2} Q^{2}+55608 \mu Q^{2}\right. \\
& \left.+28756 \mu^{2} Q+5110 \mu^{3}\right) x^{3}+4 Q^{2}\left(51173 Q^{2}+51173 Q \mu+13461 \mu^{2}\right)(Q+\mu)^{2} x^{2} \\
& \left.-69972 Q^{3}(2 Q+\mu)(Q+\mu)^{3} x+37841 Q^{4}(Q+\mu)^{4}\right)
\end{aligned}
$$

Inhomogeneous part of equation (4.27):

$$
\begin{aligned}
\mathcal{I}_{\nu}= & -\frac{1}{320 x^{11}\left(x^{2}-2 x Q+Q^{2}+x^{3}-\mu x+Q \mu\right)}\left(-33920 Q(2 Q+\mu)(Q+\mu) x^{7}\right. \\
& +64 Q(Q+\mu)\left(-1272 \mu+1919 Q \mu+1919 Q^{2}-2544 Q\right) x^{6}+64 Q(Q+\mu)\left(1844 \mu^{2}\right. \\
& \left.+11321 Q \mu-720 \mu+11321 Q^{2}-1440 Q\right) x^{5}+\left(-2528960 \mu Q^{4}+83520 \mu^{3} Q\right. \\
& -1011584 Q^{5}-5400 \mu^{4}+544704 Q^{4}-505792 \mu^{3} Q^{2}+1089408 Q^{3} \mu+628224 \mu^{2} Q^{2} \\
& \left.-2023168 \mu^{2} Q^{3}\right) x^{4}-16 Q(Q+\mu)\left(2078 \mu^{3}+44128 \mu^{2} Q-28147 \mu^{2} Q^{2}-56294 Q^{3} \mu\right. \\
& \left.+119916 \mu Q^{2}-28147 Q^{4}+79944 Q^{3}\right) x^{3}+4 Q^{2}\left(65045 \mu^{2}+370952 Q \mu\right. \\
& \left.\left.+370952 Q^{2}\right)(Q+\mu)^{2} x^{2}-423344 Q^{3}(Q+\mu)^{3}(2 Q+\mu) x+189257 Q^{4}(Q+\mu)^{4}\right)
\end{aligned}
$$


Inhomogeneous part of equation (4.29):

$$
\begin{aligned}
J_{\phi}= & -\frac{1}{16 x^{11}\left(-\mu x+x^{3}+Q^{2}-2 Q x+x^{2}+Q \mu\right)}\left(576 Q^{2}(Q+\mu)^{2} x^{6}\right. \\
& -288 Q(Q+\mu)\left(5 \mu^{2}+14 Q \mu+14 Q^{2}\right) x^{5}+\left(1080 \mu^{4}+11520 \mu^{2} Q^{3}+14400 Q^{4} \mu\right. \\
& \left.+2880 Q^{2} \mu^{3}+7488 Q^{4}+14976 \mu Q^{3}+13248 Q^{2} \mu^{2}+5760 Q \mu^{3}+5760 Q^{5}\right) x^{4} \\
& -576 Q(Q+\mu)\left(3 Q^{4}+52 Q^{3}+6 \mu Q^{3}+3 Q^{2} \mu^{2}+78 \mu Q^{2}+46 Q \mu^{2}+10 \mu^{3}\right) x^{3} \\
& +36 Q^{2}\left(1304 Q^{2}+1304 Q \mu+383 \mu^{2}\right)(Q+\mu)^{2} x^{2}-16992 Q^{3}(2 Q+\mu)(Q+\mu)^{3} x \\
& \left.+9531 Q^{4}(Q+\mu)^{4}\right)
\end{aligned}
$$

\section{B Data for charged $\kappa=0$ black holes}

Inhomogeneous parts of equations (4.30)-(4.34):

$$
\begin{aligned}
J_{A}=- & \frac{\mu^{2}}{12 x^{11}}\left(82707 Q^{3} \mu-84196 Q^{2} x \mu+32154 Q x^{2} \mu+\left(912 Q^{2}-4320 \mu\right) x^{3}-160 Q x^{4}\right) \\
J_{B}= & \frac{\mu^{2}}{8 x^{11}\left(-x \mu+Q \mu+x^{3}\right)}\left(704 Q x^{7}+\left(-11520 \mu-2304 Q^{2}\right) x^{6}+83536 Q \mu x^{5}\right. \\
& -2232 \mu\left(96 Q^{2}-5 \mu\right) x^{4}+8 Q \mu\left(26678 Q^{2}-11669 \mu\right) x^{3}+294968 Q^{2} \mu^{2} x^{2} \quad(\mathrm{~B} .2) \\
& \left.-423932 Q^{3} \mu^{2} x+210625 Q^{4} \mu^{2}\right) \\
J_{\nu}= & \frac{\mu^{2}}{320 x^{11}\left(-x \mu+Q \mu+x^{3}\right)}\left(47488 Q x^{7}-160704 Q^{2} x^{6}-132544 Q \mu x^{5}+8 \mu(3915 \mu\right. \\
& \left.+70168 Q^{2}\right) x^{4}-48 Q \mu\left(10027 Q^{2}+3274 \mu\right) x^{3}+292748 Q^{2} \mu^{2} x^{2}-349232 Q^{3} \mu^{2} x \\
& \left.+225375 Q^{4} \mu^{2}\right) \\
& \left.+26726 \mu^{2} Q x^{2}-53200 Q^{2} \mu^{2} x+31473 Q^{3} \mu^{2}\right) \\
J_{a}= & \frac{\mu}{2 x^{11}}\left(-576 Q x^{6}+3840 \mu x^{5}-16720 Q \mu x^{4}+24 \mu\left(-210 \mu+1061 Q^{2}\right) x^{3}\right.
\end{aligned}
$$




$$
\begin{aligned}
J_{\text {const }}= & -\frac{\mu^{2}}{24 x^{10}}\left(1696 Q x^{7}-3968 Q^{2} x^{6}-5656 Q \mu x^{5}+40 \mu\left(81 \mu+508 Q^{2}\right) x^{4}\right. \\
& \left.-4 Q \mu\left(5211 Q^{2}+5110 \mu\right) x^{3}+53844 Q^{2} \mu^{2} x^{2}-69972 Q^{3} \mu^{2} x+37841 Q^{4} \mu^{2}\right)
\end{aligned}
$$

Inhomogeneous part of equation (4.40):

$$
\begin{aligned}
\mathcal{I}_{\nu}= & -\frac{\mu^{2}}{320 x^{11}\left(-x \mu+Q \mu+x^{3}\right)}\left(-33920 Q x^{7}+122816 Q^{2} x^{6}+118016 Q \mu x^{5}\right. \\
& -8 \mu\left(63224 Q^{2}+675 \mu\right) x^{4}+16 Q \mu\left(28147 Q^{2}-2078 \mu\right) x^{3}+260180 Q^{2} \mu^{2} x^{2} \\
& \left.-423344 Q^{3} \mu^{2} x+189257 Q^{4} \mu^{2}\right)
\end{aligned}
$$

Inhomogeneous part of equation (4.42):

$$
\begin{aligned}
J_{\phi}= & -\frac{9 \mu^{2}}{16 x^{11}\left(-\mu x+x^{3}+Q \mu\right)}\left(1059 Q^{4} \mu^{2}-1888 Q^{3} \mu^{2} x+1532 Q^{2} \mu^{2} x^{2}+\left(-192 Q^{3} \mu\right.\right. \\
& \left.\left.-640 Q \mu^{2}\right) x^{3}+\left(320 \mu Q^{2}+120 \mu^{2}\right) x^{4}-160 Q \mu x^{5}+64 Q^{2} x^{6}\right)
\end{aligned}
$$

\section{Warp factors of the $\alpha^{\prime}$-corrected charged $\kappa=0$ black hole to leading order in $Q$}

All expressions below are to order $\mathcal{O}\left(Q^{2}\right)$ except for the gauge potential $a(x)$ which is evaluated to order $\mathcal{O}(Q)$.

Location of the horizon:

$$
x_{+}=\sqrt{\mu}-\frac{1}{2} Q
$$

Metric warp factors:

$$
\begin{gathered}
A=-\frac{60 \mu^{3}}{7 x^{6}}+\left(-\frac{4 \mu^{2}}{9 x^{5}}+\frac{5359 \mu^{3}}{112 x^{7}}\right) Q \\
B=\frac{15 \mu}{14 x^{6}}\left(37 \mu^{2}-19 x^{2} \mu-19 x^{4}\right)-\frac{Q}{504\left(\mu-x^{2}\right) x^{7}}\left(32749 \sqrt{\mu} x^{7}+103971 \mu^{4}\right. \\
\left.-136900 \mu^{3} x^{2}+180 \mu^{2} x^{4}\right)
\end{gathered}
$$




$$
\begin{aligned}
\nu= & \frac{15 \mu^{2}\left(\mu+x^{2}\right)}{32 x^{6}}+Q\left(-\frac{113241\left(2 x^{2}-\mu\right)}{5600 \mu^{3 / 2}}\left(\ln \left(1-\frac{\mu}{x^{2}}\right)+2 \operatorname{arctanh} \frac{\sqrt{\mu}}{x}\right)+\frac{9857 \mu^{3}}{3920 x^{7}}\right. \\
& \left.-\frac{1144277 \mu^{2}}{98000 x^{5}}+\frac{37747 \mu}{14000 x^{3}}-\frac{37747}{2800 x}-\frac{113241}{2800 \sqrt{\mu}}+\frac{113241 x}{1400 \mu}\right)
\end{aligned}
$$

Gauge potential:

$$
a=-\frac{193 \mu^{2}}{2 x^{4}}+\frac{835 \mu^{3}}{14 x^{6}}
$$

\section{References}

[1] J. M. Maldacena, "The large $N$ limit of superconformal field theories and supergravity," Adv. Theor. Math. Phys. 2, 231 (1998) [Int. J. Theor. Phys. 38, 1113 (1999)] [arXiv:hep-th/9711200].

[2] O. Aharony, S. S. Gubser, J. M. Maldacena, H. Ooguri and Y. Oz, Phys. Rept. 323, 183 (2000) [arXiv:hep-th/9905111].

[3] S. S. Gubser, I. R. Klebanov and A. W. Peet, "Entropy and Temperature of Black 3-Branes," Phys. Rev. D 54, 3915 (1996) [arXiv:hep-th/9602135].

[4] R. Emparan, "AdS/CFT duals of topological black holes and the entropy of zeroenergy states," JHEP 9906, 036 (1999) [arXiv:hep-th/9906040].

[5] R. G. Cai and A. z. Wang, "Thermodynamics and stability of hyperbolic charged black holes," Phys. Rev. D 70, 064013 (2004) [arXiv:hep-th/0406057].

[6] E. Witten, "Anti-de Sitter space, thermal phase transition, and confinement in gauge theories," Adv. Theor. Math. Phys. 2, 505 (1998) [arXiv:hep-th/9803131].

[7] K. Behrndt, M. Cvetic and W. A. Sabra, "Non-extreme black holes of five dimensional N = 2 AdS supergravity," Nucl. Phys. B 553, 317 (1999) [arXiv:hepth/9810227].

[8] M. Cvetic et al., "Embedding AdS black holes in ten and eleven dimensions," Nucl. Phys. B 558, 96 (1999) [arXiv:hep-th/9903214].

[9] A. Chamblin, R. Emparan, C. V. Johnson and R. C. Myers, "Charged AdS black holes and catastrophic holography," Phys. Rev. D 60, 064018 (1999) [arXiv:hepth/9902170]. 
[10] M. Cvetic and S. S. Gubser, "Phases of R-charged black holes, spinning branes and strongly coupled gauge theories," JHEP 9904, 024 (1999) [arXiv:hep-th/9902195].

[11] A. Buchel and L. A. Pando Zayas, "Hagedorn vs. Hawking-Page transition in string theory," Phys. Rev. D 68, 066012 (2003) [arXiv:hep-th/0305179].

[12] J. T. Liu and W. A. Sabra, "Mass in anti-de Sitter spaces," Phys. Rev. D 72, 064021 (2005) [arXiv:hep-th/0405171].

[13] R. G. Cai and N. Ohta, "On the thermodynamics of large N non-commutative super Yang-Mills theory," Phys. Rev. D 61, 124012 (2000) [arXiv:hep-th/9910092].

[14] S. S. Gubser, I. R. Klebanov and A. A. Tseytlin, "Coupling constant dependence in the thermodynamics of $\mathrm{N}=4$ supersymmetric Yang-Mills theory," Nucl. Phys. B 534, 202 (1998) [arXiv:hep-th/9805156].

[15] J. Pawelczyk and S. Theisen, "AdS(5) x S(5) black hole metric at O(alpha**3)," JHEP 9809, 010 (1998) [arXiv:hep-th/9808126].

[16] P. Benincasa and A. Buchel, "Transport properties of $\mathrm{N}=4$ supersymmetric YangMills theory at finite coupling," JHEP 0601, 103 (2006) [arXiv:hep-th/0510041].

[17] A. Buchel, J. T. Liu and A. O. Starinets, "Coupling constant dependence of the shear viscosity in $\mathrm{N}=4$ supersymmetric Yang-Mills theory," Nucl. Phys. B 707, 56 (2005) [arXiv:hep-th/0406264].

[18] M. T. Grisaru and D. Zanon, "Sigma Model Superstring Corrections To The Einstein-Hilbert Action," Phys. Lett. B 177, 347 (1986).

[19] M. D. Freeman, C. N. Pope, M. F. Sohnius and K. S. Stelle, "Higher Order Sigma Model Counterterms And The Effective Action For Superstrings," Phys. Lett. B 178, 199 (1986).

[20] Q. H. Park and D. Zanon, "More On Sigma Model Beta Functions And LowEnergy Effective Actions," Phys. Rev. D 35, 4038 (1987).

[21] D. J. Gross and E. Witten, "Superstring Modifications Of Einstein's Equations," Nucl. Phys. B 277, 1 (1986). 
[22] A. Buchel, "On universality of stress-energy tensor correlation functions in supergravity," Phys. Lett. B 609, 392 (2005) [arXiv:hep-th/0408095].

[23] P. Benincasa, A. Buchel and A. O. Starinets, "Sound waves in strongly coupled non-conformal gauge theory plasma," Nucl. Phys. B 733, 160 (2006) [arXiv:hepth/0507026].

[24] A. Buchel, "Transport properties of cascading gauge theories," Phys. Rev. D 72, 106002 (2005) [arXiv:hep-th/0509083].

[25] S. de Haro, A. Sinkovics and K. Skenderis, "A supersymmetric completion of the $\mathrm{R}^{* *} 4$ term in IIB supergravity," Phys. Rev. D 67, 084010 (2003) [arXiv:hepth/0210080].

[26] S. de Haro, A. Sinkovics and K. Skenderis, "On alpha' corrections to D-brane solutions," Phys. Rev. D 68, 066001 (2003) [arXiv:hep-th/0302136].

[27] G. Policastro and D. Tsimpis, "R**4, purified," arXiv:hep-th/0603165.

[28] A. Buchel, "On effective action of string theory flux compactifications," Phys. Rev. D 69, 106004 (2004) [arXiv:hep-th/0312076].

[29] O. Aharony, A. Buchel and A. Yarom, "Holographic renormalization of cascading gauge theories," Phys. Rev. D 72, 066003 (2005) [arXiv:hep-th/0506002].

[30] O. DeWolfe and S. B. Giddings, "Scales and hierarchies in warped compactifications and brane worlds," Phys. Rev. D 67, 066008 (2003) [arXiv:hep-th/0208123].

[31] R. Kallosh and A. Rajaraman, "Vacua of M-theory and string theory," Phys. Rev. D 58, 125003 (1998) [arXiv:hep-th/9805041].

[32] M. Henningson and K. Skenderis, "The holographic Weyl anomaly," JHEP 9807, 023 (1998) [arXiv:hep-th/9806087].

[33] V. Balasubramanian and P. Kraus, "A stress tensor for anti-de Sitter gravity," Commun. Math. Phys. 208, 413 (1999) [arXiv:hep-th/9902121].

[34] S. de Haro, S. N. Solodukhin and K. Skenderis, "Holographic reconstruction of spacetime and renormalization in the AdS/CFT correspondence," Commun. Math. Phys. 217, 595 (2001) [arXiv:hep-th/0002230]. 
[35] A. Buchel and J. T. Liu, "Thermodynamics of the $\mathrm{N}=2^{*}$ flow," JHEP 0311, 031 (2003) [arXiv:hep-th/0305064].

[36] A. Buchel, "N = 2* hydrodynamics," Nucl. Phys. B 708, 451 (2005) [arXiv:hepth/0406200].

[37] S. Dutta and R. Gopakumar, "On Euclidean and noetherian entropies in AdS space," arXiv:hep-th/0604070.

[38] R. M. Wald, "Black hole entropy in the Noether charge," Phys. Rev. D 48, 3427 (1993) [arXiv:gr-qc/9307038].

[39] S. Nojiri, S. D. Odintsov and S. Ogushi, "Cosmological and black hole brane world universes in higher derivative gravity," Phys. Rev. D 65, 023521 (2002) [arXiv:hep-th/0108172].

[40] M. Cvetic, S. Nojiri and S. D. Odintsov, "Black hole thermodynamics and negative entropy in deSitter and anti-deSitter Einstein-Gauss-Bonnet gravity," Nucl. Phys. B 628, 295 (2002) [arXiv:hep-th/0112045].

[41] Y. M. Cho and I. P. Neupane, "Anti-de Sitter black holes, thermal phase transition and holography in higher curvature gravity," Phys. Rev. D 66, 024044 (2002) [arXiv:hep-th/0202140].

[42] I. P. Neupane, "Black hole entropy in string-generated gravity models," Phys. Rev. D 67, 061501 (2003) [arXiv:hep-th/0212092].

[43] I. P. Neupane, "Thermodynamic and gravitational instability on hyperbolic spaces," Phys. Rev. D 69, 084011 (2004) [arXiv:hep-th/0302132].

[44] O. Aharony, J. Marsano, S. Minwalla, K. Papadodimas and M. Van Raamsdonk, "The Hagedorn / deconfinement phase transition in weakly coupled large N gauge theories," Adv. Theor. Math. Phys. 8, 603 (2004) [arXiv:hep-th/0310285].

[45] O. Aharony, J. Marsano, S. Minwalla, K. Papadodimas and M. Van Raamsdonk, "A first order deconfinement transition in large N Yang-Mills theory on a small S**3,” Phys. Rev. D 71, 125018 (2005) [arXiv:hep-th/0502149].

[46] J. O. Andersen, E. Petitgirard and M. Strickland, "Two-loop HTL thermodynamics with quarks," Phys. Rev. D 70, 045001 (2004) [arXiv:hep-ph/0302069]. 
[47] D. G. C. McKeon and A. Rebhan, "Renormalization group summation and the free energy of hot QCD," Phys. Rev. D 67, 027701 (2003) [arXiv:hep-ph/0210163].

[48] A. Fotopoulos and T. R. Taylor, "Comment on two-loop free energy in $\mathrm{N}=4 \mathrm{su}-$ persymmetric Yang-Mills theory at finite temperature," Phys. Rev. D 59, 061701 (1999) [arXiv:hep-th/9811224].

[49] M. A. Vazquez-Mozo, "A note on supersymmetric Yang-Mills thermodynamics," Phys. Rev. D 60, 106010 (1999) [arXiv:hep-th/9905030].

[50] C. j. Kim and S. J. Rey, "Thermodynamics of large-N super Yang-Mills theory and AdS/CFT correspondence," Nucl. Phys. B 564, 430 (2000) [arXiv:hepth/9905205].

[51] D. Yamada and L. G. Yaffe, "Phase diagram of $\mathrm{N}=4$ super-Yang-Mills theory with R-symmetry chemical potentials," arXiv:hep-th/0602074. 